\title{
Preview: 1999 MRS Spring Meeting
}

\section{San Francisco Marriott and Argent Hotels • San Francisco, California • April 5-9, 1999}

\author{
Meeting Chairs: \\ Katayun Barmak \\ Carnegie Mellon University \\ Paul Calvert \\ University of Arizona \\ James S. Speck \\ University of California, Santa Barbara \\ Raymond T. Tung \\ Lucent Technologies
}

\section{Technical Symposia}

The 1999 MRS Spring Meeting offers 34 technical symposia, featuring the latest developments in display materials, magnetic materials, and biological materials. The meeting will be held in the San Francisco Marriott Hotel and expand this year into the Argent (formerly ANA) Hotel, in which the display symposia will be held. The technical meeting will run from Monday, April 5 through noon Friday, April 9, and it includes 2,300 poster and oral presentations. Some of the newer topics include sensors and flat-panel displays (Symposium B), luminescent materials (E), linking materials computation and experiment $(G)$, hard and soft magnetic materials ( $\mathrm{H}$ and $\mathrm{I}$ ), patterned magnetic structures and magnetoelectronics (D), and hybrid structures (DD). While the content of the meeting is diverse, several clusters of symposia have common threads.

\section{Display Materials}

Symposia A-F are tied together by the theme of display materials. The largest components of this cluster are the symposia on Amorphous and Heterogeneous Silicon Thin Films-Fundamentals to Devices (Symposium A) and Luminescent Materials (Symposium E). Several joint sessions between Symposia $A$ and Flat Panel Displays and Sensors-Principles, Materials, and Processes (Symposium B) cover thin film transistors. Symposium B also overlaps with Symposia C, E, and F, with joint sessions planned on field emission displays, phosphors, and organic luminescent materials, respectively. Symposium C, Materials Issues in Vacuum Microelectronics II, starts Monday afternoon with a series of invited talks in a joint session with Symposium B on the status of field emission display technology, packaging, processing, applications, and reliability. Symposium F, Organic Nonlinear Optical Materials and Devices, covers photorefractive polymers, electrooptics, and other nonlinear optical behavior. The symposium on Liquid Crystal
Materials and Devices (D) starts by summarizing the effects of shape, structure, side chains and other aspects of liquid crystals and ends with new emerging technologies in areas of photofunctionality, color filters, and holography.

\section{Magnetic Materials}

The Meeting includes a group of symposia $(\mathrm{H}-\mathrm{L})$ that addresses hard and soft magnetic materials, patterned magnetic structures, magnetoelectronics, hybrid magnetic semiconductor structures, and issues related to magnetic storage media. Symposia $\mathrm{H}$ and I have several joint oral and in-room poster sessions on nanoscale hard magnetism. Symposium I (Amorphous and Nanocrystalline Materials for Hard and Soft Magnetic Applications) balances coverage of hard magnetism with ultrasoft materials. Joint sessions between I and L cover nanocrystalline magnetic thin films and thin film permanent magnets. Symposium J, Patterned Magnetic Structures and Magnetoelectronics, examines the behavior and capabilities of magnetic materials as they are shrunk to smaller dimensions. The symposium starts with a special presentation on "spintronics," which involves linking confined magnetic materials with conventional semiconductors to build exotic electronic devices. This sets the stage for subsequent sessions focused on spin and switching. The smallest symposium of the magnetism cluster, Symposium K, covers Hybrid Magnetic, Semiconductor, and Superconductor Structures, emphasizing behavior of spin and other quasiparticle degrees of freedom in confined geometries and at interfaces between dissimilar materials. Symposium K concludes Wednesday with an all-invited joint session with Symposia J and AA covering spin dynamics and transport.

\section{Silicon Devices}

Silicon-based ultralarge-scale integration (ULSI) forms the core of another cluster of symposia (M-T), addressing processing, reliability, and other aspects of microelectronic and optoelectronics research. There has been much progress recently in the reduction of defects during bulk crystal growth, the increase of the size of substrate wafers, and the improvement in wafer surface morphology for technologically important semiconducting and insulating materials. These developments as well as the latest developments in wafer bonding, silicon-on-insulator (SOI) structures, and compliant substrates form the basis of a new symposium, Advanced Semiconductor Wafer Engineering (T). Other symposia in this cluster cover a broad spectrum of the processes needed to make devices, such as front-end doping in silicon (S), ultraclean processing (Q), chemicalmechanical polishing $(P)$, low- and highdielectric constant materials ( $O$ and $R$ ) including ultrathin $\mathrm{SiO}_{2}$ films, materials reliability $(\mathrm{M})$, and advanced interconnects and contacts $(\mathrm{N})$. Many joint sessions bring into focus the connections between these symposia.

\section{Biological Materials}

A cluster of five symposia (DD-HH) have connections to medical, biological, and other organic materials. Symposium FF, Biomedical Materials, covers materials for soft and hard tissue implants and tissue engineering. Symposium EE deals with polymers produced by biotechnology, including fermentation, polymers for tissue culture and cell adhesion and for controlled drug release. Membranes, Symposium GG, will cover polymer, inorganic, and biological membranes with a considerable emphasis on lipid membranes. Soft Condensed Matter-Fundamentals and Applications (Symposium $\mathrm{HH}$ ) addresses gels and concentrated particle suspensions, including virus and cell suspensions. Several sessions address electrorheological fluids. In addition Organic-Inorganic Hybrids, Symposium DD, will include selfassembled materials and hybrids with entrained proteins and cells.

\section{Continuing Topics}

Various aspects of semiconductors, particularly compound and more complex semiconductors can be found in Symposia U-BB.

On a fundamental level, Epitaxial Growth-Principles and Applications is covered in Symposium V. This symposium is dedicated to the memory of Mohan Krishnamurthy, who has made nominal contributions to the field and was one of the original organizers for this symposium.

Semiconductor Quantum Dots (Symposium $W$ ) continue to gain attention. Dots made of $\mathrm{Si}, \mathrm{Ge}$, compound semiconductors, and self-assembled organics will be considered, including how to grow and characterize them. This symposium also includes a presentation by Chad A. Mirkin (Northwestern University), the 1999 MRS Outstanding Young Investigator, on DNA-Based Methodology for Preparing 
Nanocluster Circuits and Arrays.

Symposium $Y$, Wide-Bandgap Semiconductors for High-Power, High-Frequency, and High-Temperature Applications, includes a panel discussion 8:00 p.m. Wednesday evening on Wide-Bandgap Semiconductor Products featuring speakers from Cree Research, Northrop Grumman, Siemens, Honeywell, Rockwell, and HRL Laboratories.

An important issue in the use of compound semiconductors is surface passivation and finding processing methods that work for these somewhat complex materials. Symposium $\mathrm{Z}$ examines these issues.

Symposium BB explores Multicomponent Oxide Films for Electronics. Exceptional properties of multicomponent oxides, combined with the inability of simpler materials to meet the increasing demands of the electronics industry, have motivated tremendous interest and activity in using multicomponent oxides films for electronic applications. This symposium focuses on processing and characterization and their contributions to device applications. Epitaxial multicomponent oxide film growth is covered in a joint session with Symposium A.

Symposium AA examines the technique of Near-Field Scanning Optical Microscopy and Spectroscopy. The symposium addresses its application to semiconductors, organics, quantum structures. As such, several joint sessions are scheduled, for instance to cover near-field spectroscopy of quantum dots, wires, and metals.

Symposium U, In-Situ Process Diagnostic and Modeling, incorporates electron and ion beam analysis, ellipsometry and optical characterization, electron and probe microscopy, and other related techniques.

It has become increasingly possible to link materials computation and experiment, and Symposium $G$ provides a forum for doing just that. By examining transport, defects, interfacial thermodynamics, and mechanical properties using both experimental and computational approaches, results can be validated or directions for further research can be more easily targeted.

Yet another area covered during this meeting is New Materials for Batteries and Fuel Cells (Symposium CC), covering cathodes, proton exchange membrane fuel cells, polymer electrolytes, supercapacitors, and solid oxide conductors.

\section{Special Events}

During the Monday evening awards ceremony, April 5, beginning at 6:00 p.m. in the Marriott, MRS will present the Outstanding Young Investigator Award to Chad A. Mirkin, Morrison Professor of Chemistry, Northwestern University, and the Gold and Silver Graduate Student Awards to graduate students who authored or co-authored symposium papers which exemplified significant and timely research. Graduate Student Award Finalists' Special Talk Sessions will be held that Monday beginning at noon. The Plenary address by Paolo Gargini (Intel) follows the awards ceremony.

Symposium X, Frontiers of Materials Research, presents a series of authoritative reviews for the nonspecialist at noontime, Monday through Thursday. Topics parallel those of the technical symposia. The latest developments in silicon crystal growth and key materials for the next generation of integrated devices will be presented, reflecting the theme of silicon ULSI. Ferroelectrics are covered in two talks, one on process sensitivity and materials design for ferroelectric memories and the other on adaptive-learning neurochips. In step with the biological emphasis of this meeting, a talk addresses the use of genes and proteins to control biological nanofabrication with silica. Another goes on wings of flies, and examines aerodynamics and neurobiology of fly flight. Another presentation looks at Cryogenic Microcalorimeters for Next Generation X-Ray Detectors for Microchemical Analysis.
Poster Sessions, held Tuesday and Wednesday evenings, include a competition for the best posters. Awards of $\$ 500$ will be given to the presenting author of the most outstanding poster(s) as selected by the Meeting Chairs at each evening's session. Posters will be judged on their technical content, appearance, graphic excellence, and presentation quality.

A major Exhibit encompassing a full spectrum of equipment, instrumentation, products, software, publications, and services will be held Tuesday through Thursday in the Marriott. Meeting participants are invited to attend a reception on Tuesday evening from 5:00-6:30 p.m. in the exhibit area.

All graduate students and members of MRS University Chapters are invited to attend the Student Mixer reception (date, time, and location to be announced in the Meeting Guide on site). Also, University Chapter officers and faculty advisors are invited to attend a meeting of MRS University Chapter representatives to compare notes on recent activities and brainstorm on new projects and issues of common concern.

A Job Center for MRS meeting attendees will be open Tuesday through Thursday, 9:00 a.m-5:00 p.m., in the Marriott Hotel.

See the following pages for a matrix of symposia sessions, highlights of special events including five tutorials and a National Science Foundation Seminar on materials research support, profiles of exhibitors, and hotel and transportation arrangements. The 1999 Spring Meeting program is available on the MRS Website (www.mrs.org). For additional information regarding any of the meeting activities, contact MRS Member Services, 506 Keystone Drive, Warrendale, PA 15086-7573; e-mail: info@mrs.org; fax 724-779-8313; phone 724779-3003. The deadline to preregister for the meeting is Friday, March 19.

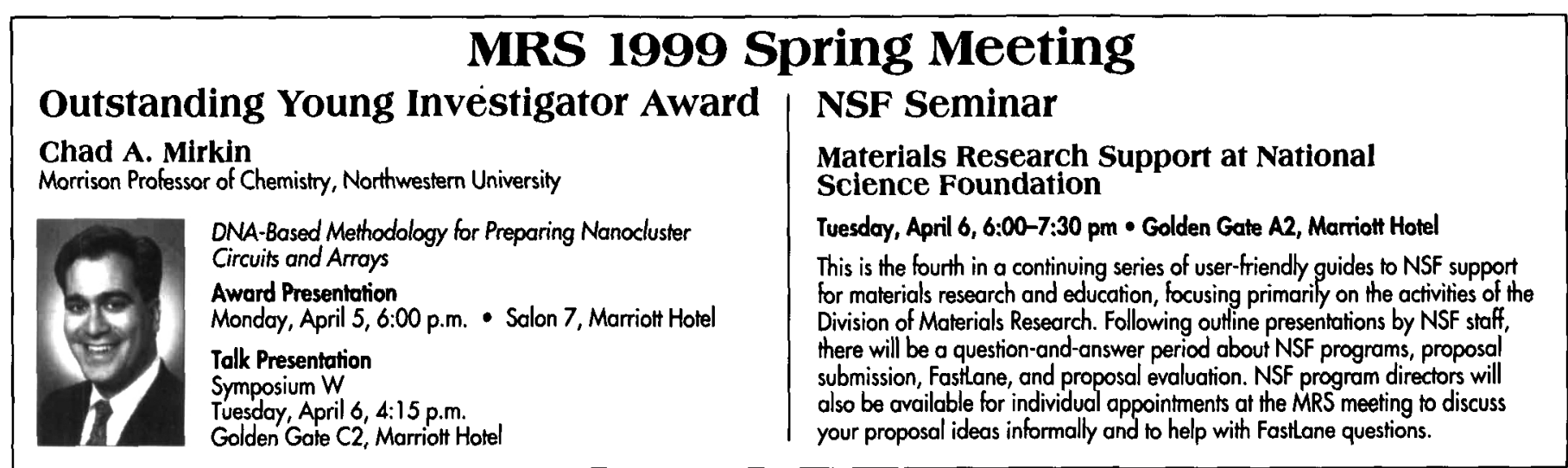




\section{MRS 1999 Spring Meeting • Lodging \& Travel}

\section{Hotel Reservations}

A block of rooms has been reserved for MRS meeting attendees at the San Francisco Marriott and Argent (formerly ANA) Hotels, 30 minutes from the San Francisco International Airport. When making your reservation, mention the Materials Research Society to receive the special rate. A Hotel Reservation Form is available on the MRS Web site (wuw.mrs.org) and in the Program Book.

Deadline for hotel reservations: March 1, 1999 Rooms are limited-reserve yours early!

\section{San Francisco Marriott Hotel}

55 Fourth Street

San Francisco, CA 94103

Phone Reservations: $415-442-6755$

Fax Reservations: $\quad 415-442-0141$

Rate: $\bullet \$ 135 /$ Single

- \$155/Double (plus 14\% City Tax)

Argent Hotel (formerly ANA)

50 Third Street

San Francisco, CA 94103

Phone Reservations: $415-974-6400$

Fax Reservations: $\quad 415-974-8820$

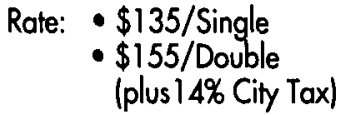

To contact hotel guests:

Marriolt: Tel 415-896-1600; Fax 415-777-2799

Argent: Toll Free (US \& Canada) 877-222-6699

Tel 415-974-6400; Fax 415-543-8268

\section{Airline Transportation}

Special, discounted air fares have been arranged as a service to MRS Spring Meeting attendees. Refer to the MRS Web site (www.mrs.org) or the Program Book for the Discount Air Fare Form.

\section{Local Transportation}

The San Francisco Airporter service is available between the airport and downtown San Francisco hotels.

\section{Parking}

Parking is available at the Marriott and Argent Hotels and nearby public facilities.

\section{Child Care}

Check with the Concierge Desks for a comprehensive roster of licensed and bonded sitters.

\section{Symposium Tutorials}

(Details available on the MRS Web site and in the Program Book)

\section{Sunday • April 4}

\section{Symposium B}

STB: Flat Panel Display Technologies

2:00 pm - 5:00 pm

Salon 4-6 - Marriott

\section{Monday • April 5}

\section{Symposium A}

STA: Amorphous Silicon Materials and Devices for Large-Area Electronics

$8: 30 \mathrm{am}-4: 30 \mathrm{pm}$

Metroplitan III - Argent

\section{Symposium $\mathbf{H}$}

STH: Concepts and Experimental Methods in Micromagnetism

2:00 pm-5:00 pm

Salon 6-Marriolt

\section{Symposium 0}

STO: Low-Dielectric Constant Materials for B.E.O.L. High-Performance Integrated Circuits 1:30 pm - 5:00 pm Golden Gate A1 - Marriott
Tuesday • April 6

\section{Symposium BB}

STb: Mutticomponent Oxide Thin Film Fabrication Techniques-PLD, Sputtering, MOCVD, and MBE Methods

$8: 30 \mathrm{am}-11: 45 \mathrm{am}$

Salon 7 - Marriolt 


\section{MRS 1999 Spring Meeting • Session Locator}

\begin{tabular}{|c|c|c|c|c|c|c|c|c|}
\hline & \multirow[b]{2}{*}{ SYMPOSIUM } & \multirow[b]{2}{*}{ LOCATION } & \multicolumn{3}{|c|}{ MONDAY, APRIL 5} & \multicolumn{3}{|c|}{ TUESDAY, APRIL 6} \\
\hline & & & a.m. & p.m. & eve. & a.m. & p.m. & eve. \\
\hline A: & $\begin{array}{l}\text { Amorphous and Hetero- } \\
\text { geneous Silicon Thin Films }\end{array}$ & Metropolitan III & Tutorial Session" & Tutorial Session" & & $\begin{array}{l}\text { A1: Growth Mechanism } \\
\text { A2: Metastability }\end{array}$ & $\begin{array}{l}\text { A3/B5: Amorphous and } \\
\text { Heterogeneous TFTs } \\
\text { A4/B6: TFTs and Displays }\end{array}$ & $\begin{array}{r}\text { A5-A11: } \\
\text { Posters } \\
\text { (A) } \\
\end{array}$ \\
\hline B: & $\begin{array}{l}\text { Flat Panel Displays \& Sensors } \\
\text { Sunday Tutorial Session* } \\
\text { SALON } 4-6 \text { (M) }\end{array}$ & $\begin{array}{l}\text { Metropolitan I } \\
\text { (A) }\end{array}$ & $\begin{array}{l}\text { B1/E1: Inorganic Low-Voltage } \\
\text { Phosphors } \\
\text { B2/E2: Thin Film Phosphors }\end{array}$ & $\begin{array}{l}\text { B3/C1: Field Emission } \\
\text { Displays }\end{array}$ & & $\begin{array}{l}\text { B4/C2: Field Emission Devices } \\
\text { and Displays }\end{array}$ & $\begin{array}{l}\text { B5/A3: Amorphous and } \\
\text { Heterogeneous TFTs } \\
\text { B6/A4: TFTs and Displays } \\
\text { METROPOLITAN III }\end{array}$ & $\begin{array}{l}\text { B7/CA, B8: } \\
\text { Posters } \\
\text { (A) }\end{array}$ \\
\hline c: & $\begin{array}{l}\text { Materials Issues in Vacuum } \\
\text { Microelectronics II }\end{array}$ & $\begin{array}{l}\text { Franciscan III } \\
\text { (A) }\end{array}$ & & $\begin{array}{l}\text { C1/B3: Field Emission } \\
\text { Displays } \\
\text { MEIROPOLITAN I }\end{array}$ & & $\begin{array}{l}\text { C2/B4: Field Emission Devices } \\
\text { and Displays } \\
\text { METROPOLITANI }\end{array}$ & $\begin{array}{l}\text { C3: Novel Fabrication and } \\
\text { Materials for Field Emitters }\end{array}$ & \begin{tabular}{|l} 
C4/B7: \\
Posters \\
$\quad$ (A) \\
\end{tabular} \\
\hline D: & $\begin{array}{l}\text { Liquid Crystal Materiais and } \\
\text { Devices }\end{array}$ & $\begin{array}{l}\text { Concordia } \\
\text { (A) }\end{array}$ & D1: New Materials and Effects & $\begin{array}{l}\text { D2: Polymer/Liquid Crystal } \\
\text { Composites }\end{array}$ & $\begin{array}{l}\text { D3: New Matis. } \\
\text { and Effects }\end{array}$ & $\begin{array}{l}\text { D4: Liquid Crystalline Polymers } \\
\text { and Applications }\end{array}$ & \begin{tabular}{l|} 
D5: Display Technologies \\
and Modeling
\end{tabular} & $\begin{array}{l}\text { D6: Posters } \\
\text { (A) }\end{array}$ \\
\hline E: & Luminescent Materials & $\begin{array}{c}\text { Franciscan I } \\
\text { (A) }\end{array}$ & $\begin{array}{l}\text { E1/B1: Inorganic Low-Voltage } \\
\text { Phosphors } \\
\text { E2/B2: Thin-Film Phosphors } \\
\text { METROPOLITAN I } \\
\end{array}$ & E3: Inorganic Phosphors & & $\begin{array}{l}\text { E4: Synthesis, Processing and } \\
\text { Characterization }\end{array}$ & E5: Mechanisms and Defects & $\begin{array}{l}\text { E6: Posters } \\
\text { (A) }\end{array}$ \\
\hline $\mathrm{F}:$ & $\begin{array}{l}\text { Organic Nonlinear Optical } \\
\text { Materials and Devices }\end{array}$ & $\begin{array}{l}\text { Franciscan II } \\
\text { (A) }\end{array}$ & & & & F1: Nonlinear Optical Materials & F2: Electro-Optics & $\begin{array}{l}\text { F3: Posters } \\
\text { (A) }\end{array}$ \\
\hline G: & $\begin{array}{l}\text { Linking Materials Computation } \\
\text { and Experiment }\end{array}$ & $\begin{array}{l}\text { City } \\
\text { (A) }\end{array}$ & $\begin{array}{l}\text { G1: Methods/Transport } \\
\text { Properties }\end{array}$ & $\begin{array}{l}\text { G2: Process Modeling/Point } \\
\text { and Line Defects }\end{array}$ & & $\begin{array}{l}\text { G3: Interfacial Structure and } \\
\text { Thermodynamics }\end{array}$ & G4: Interfacial Dynamics & \\
\hline $\mathrm{H}:$ & Advanced Hard Magnets & $\begin{array}{l}\text { Salon } 6 \\
\text { (M) }\end{array}$ & & Tutorial Session & & $\begin{array}{l}\text { H1: Permanent Magnet } \\
\text { Processing }\end{array}$ & $\begin{array}{l}\text { H2: Intrinsic Properties of } \\
\text { Permanent Magnetic } \\
\text { Materials }\end{array}$ & \\
\hline : & $\begin{array}{l}\text { Amorphous and Nanocrys- } \\
\text { talline Materials for Hard and } \\
\text { Soft Magnetic Applications }\end{array}$ & $\begin{array}{l}\text { Salon } 5 \\
\text { (M) }\end{array}$ & 11: Fine Particle Magnets & $\begin{array}{l}\text { 12: Nanocrystalline Antiferro- } \\
\text { and Ferrimagnets }\end{array}$ & & $\begin{array}{l}\text { 13: Ultrasoft Nanocrystalline and } \\
\text { Amorphous Materials I }\end{array}$ & $\begin{array}{l}\text { 14: Uttrasofi Nanocrystalline } \\
\text { and Amorphous Materials II }\end{array}$ & $\begin{array}{l}\text { 15: Posters } \\
\text { (M) }\end{array}$ \\
\hline J: & $\begin{array}{l}\text { Patterned Magnetic } \\
\text { Structures and } \\
\text { Magnetoelectronics }\end{array}$ & $\mathrm{Salon} 3_{(\mathrm{M})}$ & $\begin{array}{l}\text { J1: Spintronics } \\
\text { J2: Spin Magnetoelectronics }\end{array}$ & $\begin{array}{l}\text { J3: Novel Fabrication } \\
\text { Methods I I Fabrication } \\
\text { J4: Novel Fabrion } \\
\text { Methods II } \\
\end{array}$ & & $\begin{array}{l}\text { 55: Characterization and } \\
\text { Magnetic Switching } \\
\text { J6: Magnetic Switching }\end{array}$ & \begin{tabular}{|l|l} 
J7: Magnetic Switching \\
Speed \\
J8: Magnetic Nano-Elements \\
and Domain Walls
\end{tabular} & $\begin{array}{c}\text { J9: Posters } \\
(\mathrm{M})\end{array}$ \\
\hline K: & $\begin{array}{l}\text { Hybrid Magnetic, } \\
\text { Semiconductor, and } \\
\text { Superconductor Structures }\end{array}$ & $\begin{array}{l}\text { Salon } 2 \\
\text { (M) }\end{array}$ & $\begin{array}{l}\text { K1: Hybrid Superconductor } \\
\text { Structures }\end{array}$ & $\begin{array}{l}\text { K2: Ferromagnet- } \\
\text { Semiconductor Devices } \\
\text { and Spin Transport }\end{array}$ & & $\begin{array}{l}\text { K3: Semiconductor/Magnetic } \\
\text { Compound Heterostructures }\end{array}$ & \begin{tabular}{l|l|} 
K4: Magnetic & \\
Semiconductors
\end{tabular} & $\begin{array}{c}\text { K5: Posters } \\
\text { (M) }\end{array}$ \\
\hline L: & $\begin{array}{l}\text { Polycrystalline Metal and } \\
\text { Magnetic Thin Films }\end{array}$ & $\begin{array}{l}\text { Salon } 4 \\
(M)\end{array}$ & & L1: Magnetic Thin Films & & $\begin{array}{l}\text { L2: Textured and Multilayered } \\
\text { Thin Films }\end{array}$ & \begin{tabular}{|l|} 
L3/N4: Copper \\
Microstructure \\
GOLDEN GATE B2
\end{tabular} & \\
\hline M: & $\begin{array}{l}\text { Materials Reliability in } \\
\text { Microelectronics IX }\end{array}$ & $\begin{array}{l}\text { Salon } 10 \\
\text { (M) }\end{array}$ & & & & $\begin{array}{l}\text { M1/R4: Reliability of Ultrathin } \\
\text { Gate Dielectrics } \\
\text { NOB HILL B/C/D } \\
\end{array}$ & $\begin{array}{l}\text { M2: Solder and Barrier Layer } \\
\text { Reliability }\end{array}$ & \\
\hline $\mathrm{N}:$ & $\begin{array}{l}\text { Advanced Interconnects and } \\
\text { Contacts }\end{array}$ & $\begin{array}{l}\text { Goiden Gate B2 } \\
\text { (M) }\end{array}$ & $\begin{array}{l}\text { N1: Silicides-Titanium and } \\
\text { Cobalt Silicide }\end{array}$ & N2: Cobalt and Nickel Silicide & & $\begin{array}{l}\text { N3: Copper Thin Films and } \\
\text { Integration }\end{array}$ & $\begin{array}{l}\text { N4/L3: Copper } \\
\text { Microstructure } \\
\end{array}$ & $\begin{array}{l}\text { N5: Posters } \\
(\mathrm{M})\end{array}$ \\
\hline o: & $\begin{array}{l}\text { Low-Dielectric Constant } \\
\text { Materiais \& Applications in } \\
\text { Microelectronics }\end{array}$ & $\begin{array}{l}\text { Golden Gate A1 } \\
\text { (M) }\end{array}$ & & Tutorial Session" & & $\begin{array}{l}\text { O1: Porous Films-Organic and } \\
\text { Inorganic }\end{array}$ & $\begin{array}{l}\text { O2: Porous Films- } \\
\text { Inorganic/Low-k Integration }\end{array}$ & \\
\hline P: & $\begin{array}{l}\text { Chemical-Mechanical } \\
\text { Polishing }\end{array}$ & $\begin{array}{c}\text { Nob Hill A } \\
(M)\end{array}$ & $\begin{array}{l}\text { P1: Overview and Oxide } \\
\text { Polishing }\end{array}$ & P2: Pads and Related Issues & & P3: Metal Polishing - W and AL & $\begin{array}{l}\text { P4: Copper Polishing and } \\
\text { Related Issues }\end{array}$ & \\
\hline Q: & $\begin{array}{l}\text { Ultraclean Processing of } \\
\text { Semiconductor Structures } \\
\text { and Devices }\end{array}$ & $\begin{array}{c}\text { Nob Hill B } \\
(M)\end{array}$ & & & & & & \\
\hline R: & $\begin{array}{l}\text { Ultrathin } \mathrm{SiO}_{2} \text { and High-k } \\
\text { Materials for ULSI Gate } \\
\text { Dielectrics }\end{array}$ & $\begin{array}{l}\text { Nob Hill } B / C / D \\
\text { (M) }\end{array}$ & $\begin{array}{l}\text { R1: Advances in Ultrathin } \\
\text { Oxides and Oxynitidides I } \\
\text { R2: Silicon Nitride I }\end{array}$ & $\begin{array}{l}\text { R3: Atomic Scale Control of the } \\
\text { Dielectric/Silicon Interface }\end{array}$ & & $\begin{array}{l}\text { R4/M1: Reliability of Ultrathin } \\
\text { Gate Dielectrics }\end{array}$ & $\begin{array}{l}\text { R5: Electrical Properties of } \\
\text { Ultrathin Gate Dielectrics }\end{array}$ & $\begin{array}{l}\text { R6: Posters } \\
\text { (M) }\end{array}$ \\
\hline S: & Si Front-End Processing & $\begin{array}{l}\text { Golden Gate C1 } \\
\text { (M) }\end{array}$ & & & & S1: Ultra-Shallow functions I & $\begin{array}{l}\text { S2: Ultra-Shallow Junctions II } \\
\text { S3/T5: Mechanisms of Point. } \\
\text { Defect Interaction \& Diffusion }\end{array}$ & \\
\hline $\mathrm{T}:$ & $\begin{array}{l}\text { Advanced Semiconductor } \\
\text { Wafer Engineering }\end{array}$ & $\begin{array}{l}\text { Golden Gate C3 } \\
\text { (M) }\end{array}$ & T1: Silicon Defect Engineering & T2: Silicon Gettering/GaAs & & T3: Silicon Carbide and Simox & $\begin{array}{l}\text { T4: Wafer Bonding I } \\
\text { T5/S3: Mechanisms of Point- } \\
\text { Defect Interaction \& Diffusion } \\
\text { GOLDEN GATE C1 }\end{array}$ & \\
\hline U: & $\begin{array}{l}\text { In-Situ Process Diagnostic } \\
\text { and Modeling }\end{array}$ & $\begin{array}{l}\text { Golden Gate A3 } \\
\text { (M) }\end{array}$ & & & & $\begin{array}{l}\text { U1: In Situ lon-and Electron- } \\
\text { Beam Analysis }\end{array}$ & \begin{tabular}{l|} 
U2: In Situ Spectroscopic \\
Ellipsometry and Other \\
Optical Characterization \\
\end{tabular} & $\begin{array}{l}\text { U3: Posters } \\
\text { (M) }\end{array}$ \\
\hline V: & $\begin{array}{l}\text { Epitaxial Growth-Principles } \\
\text { and Applications }\end{array}$ & $\begin{array}{l}\text { Golden Gate B3 } \\
\text { (M) }\end{array}$ & $\begin{array}{l}\text { V1: Submonolayer and } \\
\text { Multilayer Growh }\end{array}$ & V2: Characterization & & V3: Strained Systems I & V4: Strained Systems II & \\
\hline W: & Semiconductor Quantum Dots & $\begin{array}{l}\text { Golden Gate C2 } \\
\text { (M) }\end{array}$ & W1: Si and Ge Dots & $\begin{array}{l}\text { W2: II-VI and Other Free- } \\
\text { Standing (Colloidal) Dots }\end{array}$ & & $\begin{array}{l}\text { W3/AA3: Near-Field Spectros- } \\
\text { copy of Quantum Dots, Wires } \\
\text { and Metals }\end{array}$ & $\begin{array}{l}\text { W4: Organiz.Dots/Dot Arrays } \\
\text { W5: Biological \& Molec. Syst. } \\
4: 15 \text { Outstanding Young } \\
\text { Investigator's Talk } \\
\end{array}$ & \\
\hline$x:$ & $\begin{array}{l}\text { Frontiers of Materials } \\
\text { Research }\end{array}$ & $\begin{array}{c}\text { Salon } 7 \\
(M)\end{array}$ & & $x 1$ & & & $x_{2}$ & \\
\hline$Y:$ & $\begin{array}{l}\text { Wide-Bandgap Semiconduc- } \\
\text { tors for High-Power, High- } \\
\text { Frequency, and High- } \\
\text { Temperature Applications }\end{array}$ & $\begin{array}{l}\text { Golden Gate A2 } \\
\text { (M) }\end{array}$ & $\begin{array}{l}\text { Y1: SiC Devices and } \\
\text { Processing I }\end{array}$ & $\begin{array}{l}\text { Y2: SiC Epitaxial Growth and } \\
\text { Characterization }\end{array}$ & & $\begin{array}{l}\text { Y3: SiC Bulk Growth and } \\
\text { Characterization }\end{array}$ & $\begin{array}{l}\text { Y4: SiC Devices and } \\
\text { Processing II }\end{array}$ & $\begin{array}{l}\text { Y5: Posters } \\
\text { (M) }\end{array}$ \\
\hline Z: & $\begin{array}{l}\text { Compound Semiconductor } \\
\text { Surface Passivation \& Novel } \\
\text { Device Processing }\end{array}$ & $\begin{array}{l}\text { Golden Gate B1 } \\
\text { (M) }\end{array}$ & & $\begin{array}{l}\text { Z1: Fundamentals of Surfaces } \\
\text { and Their Passivation }\end{array}$ & & $\begin{array}{l}\text { 22: Novel Approaches for } \\
\text { Surface Passivation and } \\
\text { Device Processing }\end{array}$ & $\begin{array}{l}\text { 23: Oxides-Structural, } \\
\text { Transport, and Optical } \\
\text { Properties }\end{array}$ & $\begin{array}{l}\text { Z4: Posters } \\
\text { (M) }\end{array}$ \\
\hline & $\begin{array}{l}\text { Near-Field Scanning Optical } \\
\text { Microscopy \& Spectroscopy }\end{array}$ & $\begin{array}{l}\text { Salon } 1 \\
\text { (M) }\end{array}$ & $\begin{array}{l}\text { AA1: Semiconductor } \\
\text { Heterostructures }\end{array}$ & $\begin{array}{l}\text { AA2: Thin Films, Polymers, and } \\
\text { Molecules }\end{array}$ & & $\begin{array}{l}\text { AA3/W3: Near-Field Spectros- } \\
\text { copy of Quantum Dots, Wires \& } \\
\text { Metals GOLDEN GATE C2 }\end{array}$ & AA4: Proteins and Polymers & \\
\hline & $\begin{array}{l}\text { Multicomponent Oxide Films } \\
\text { for Electronics }\end{array}$ & $\begin{array}{l}\text { Salon } 7 \\
(\mathrm{M})\end{array}$ & & & & Tutorial Session & $\begin{array}{l}\text { BB1: Properties of Multi- } \\
\text { component Oxides }\end{array}$ & $\begin{array}{l}\mathrm{BB2}, \mathrm{BB3}: \\
\text { Posters (M) }\end{array}$ \\
\hline & $\begin{array}{l}\text { Now Materials for Batteries } \\
\text { and Fuel Cells }\end{array}$ & $\begin{array}{l}\text { Metropolitan II } \\
\text { (A) }\end{array}$ & CC1: Cathodes I & CC2: Cathodes II & & CC3: PEM Fuel Cells & $\begin{array}{l}\text { CC4: PEM Fuel Cells' } \\
\text { Polymer Electrolytes }\end{array}$ & $\begin{array}{l}\text { CC5: Posters } \\
\text { (A) }\end{array}$ \\
\hline & $\begin{array}{l}\text { Organic-Inorganic Hybrid } \\
\text { Materials }\end{array}$ & $\begin{array}{l}\text { Salon } 11 / 12 \\
(M)\end{array}$ & $\begin{array}{l}\text { DD1: Synthesis and } \\
\text { Processing I }\end{array}$ & $\begin{array}{l}\text { DD2: Synthesis and } \\
\text { Processing II }\end{array}$ & & \begin{tabular}{|l|} 
DD3: Novel Ordered Hybrid \\
Structures \\
DD4: Structure and Properties \\
of Organic Monolayers \\
\end{tabular} & $\begin{array}{l}\text { DD5: Nonsilicate Hybrid } \\
\text { Materials }\end{array}$ & \\
\hline & Polymers in Biotechnology & $\begin{array}{l}\text { Salon } 15 \\
\text { (M) }\end{array}$ & $\begin{array}{l}\text { EE1: Hydrogel Systems } \\
\text { EE2: Tissue Engineering } \\
\text { Applications }\end{array}$ & $\begin{array}{l}\text { EE3: Biopolymers \& Biological } \\
\text { Production of Polymers } \\
\text { EE4: Polymers at Surfaces } \\
\end{array}$ & & $\begin{array}{l}\text { EE5: Drug Delivery Applications } \\
\text { EE6: Stimuli-Responsive and } \\
\text { Bioactive Systems }\end{array}$ & & \\
\hline & Biomedical Materials & $\begin{array}{c}\text { Salon } 15 \\
(\mathrm{M})\end{array}$ & & & & & & \\
\hline & : Membranes & $\begin{array}{l}\text { Salon } \\
\text { (M) }\end{array}$ & & & & GG1: Inorganic Membranes & GG2: Polymer Membranes & \\
\hline & A: Soft Condensed Matter & $\begin{array}{c}\text { Salon } 13 \\
(\mathrm{M})\end{array}$ & HH1: Gels & $\begin{array}{l}\mathrm{HH} 2: \text { Gels and Spherical } \\
\text { Colloids } \\
\end{array}$ & & $\begin{array}{l}\text { HH3: Magnetorheological } \\
\text { Fluids/Electrorheological Fluids } \\
\end{array}$ & $\begin{array}{l}\text { HH4: Electrorheological } \\
\text { Fluids/Suspensions of Rods }\end{array}$ & \\
\hline
\end{tabular}




\section{MRS 1999 Spring Meeting • Session Locator}

\begin{tabular}{|c|c|c|c|c|c|c|c|}
\hline \multicolumn{3}{|c|}{ WEDNESDAY, APRIL 7} & \multicolumn{3}{|c|}{ THURSDAY, APRIL 8} & \multicolumn{2}{|c|}{ FRIDAY, APRIL 9} \\
\hline a.m. & p.m. & eve. & a.m. & p.m. & eve. & a.m. & p.m. \\
\hline $\begin{array}{l}\text { A12: Heterogeneous Materials I } \\
\text { A13: Hydrogen }\end{array}$ & $\begin{array}{l}\text { A14: Hot-Wire Deposition } \\
\text { A15: Solar Cells }\end{array}$ & $\begin{array}{l}\text { A16-A20: } \\
\text { Posters } \\
\text { (A) }\end{array}$ & $\begin{array}{l}\text { A21: Detectors \& Novel Devices } \\
\text { A22: Defects, Banctails \& } \\
\text { Transport }\end{array}$ & $\begin{array}{l}\text { A23: Heterogeneous Matts. II } \\
\text { A24: Ordering \& } \\
\text { Protocrystallinity }\end{array}$ & & $\begin{array}{l}\text { A25: Hydrogen In Metastability } \\
\text { A26: High Deposition Rate }\end{array}$ & \\
\hline $\begin{array}{l}\text { B9: AMLCDI } \\
\text { B10: AMLCD II }\end{array}$ & $\begin{array}{l}\text { B11: Large-Area Processes } \\
\text { B12: Sensors } \\
\text { B13/EB/FG: Organic Lum. Matls. } \\
\text { FAANCISCAN I }\end{array}$ & $\begin{array}{l}\text { B14: Posters } \\
\text { (A) }\end{array}$ & B15/F8: OLEDs & $\begin{array}{l}\text { B16/F9: Electroluminescent } \\
\text { Materials }\end{array}$ & & B17/F10: Organic ELI & \\
\hline $\begin{array}{l}\text { C5: Carbon and Diamond Field } \\
\text { Emitters }\end{array}$ & $\begin{array}{l}\text { C6: Theory and Modeling of } \\
\text { Electron Field Emission }\end{array}$ & & & & & & \\
\hline \multicolumn{8}{|l|}{$\begin{array}{l}\text { D7: New Emerging } \\
\text { Technologies }\end{array}$} \\
\hline E7: Novel Materials & $\begin{array}{l}\text { E8/B13/F6: Organic } \\
\text { Luminescent Materials }\end{array}$ & & $\begin{array}{l}\text { E9: Theory and Luminescence } \\
\text { Sensors }\end{array}$ & & & & \\
\hline F4: Photorefractive Polymers & $\begin{array}{l}\text { F5: Electronic \& Optical Proc. } \\
\text { F6/813/E8: Organic Lum. Matls. } \\
\text { FRANGISCAN I }\end{array}$ & $\begin{array}{l}\text { F7: Posters } \\
\text { (A) }\end{array}$ & $\begin{array}{l}\text { F8/B15: OLEDs } \\
\text { METROPOLITAN I }\end{array}$ & $\begin{array}{l}\text { F9/B16: Electroluminescent } \\
\text { Materials } \\
\text { METROPOLITAN I }\end{array}$ & & $\begin{array}{l}\text { F10V17: Organic ELI } \\
\text { METROPOLITAN I }\end{array}$ & \\
\hline G5: Mechanical Properties & G6: Atomistic-Level Simulations & $\begin{array}{l}\text { G7: Posters } \\
\text { (A) }\end{array}$ & & & & & \\
\hline $\begin{array}{l}\text { H3/16: Nanoscale Hard } \\
\text { Magnetism I } \\
\text { SALON 5/6 }\end{array}$ & $\begin{array}{l}\text { H4/17: Nanoscale Hard } \\
\text { Magnetism II } \\
\text { H5AL: Posters } \\
\text { SALON } 5 / 6\end{array}$ & & $\begin{array}{l}\text { H6: Permanent Magnet } \\
\text { Applications } \\
\text { H7: Microstructure and } \\
\text { Micromagnetics }\end{array}$ & $\begin{array}{l}\text { H8: Microstruc/ Micromagnet. } \\
\text { H9/10/L8: Thin-Film Permanent } \\
\text { Magnets } \\
\text { SALON 1-3 }\end{array}$ & & & \\
\hline $\begin{array}{l}\text { 16/H3: Nanoscale Hard } \\
\text { Magnetism I } \\
\text { SALON } 5 / 6\end{array}$ & $\begin{array}{l}\text { 17/H4: Nanoscale Hard } \\
\text { Magnetism II } \\
\text { 18//55: Posters SALON 5/6 }\end{array}$ & & $\begin{array}{l}\text { 19/L7: Nanocrystalline Magnetic } \\
\text { Thin Films } \\
\text { SALON } 4 / 5\end{array}$ & $\begin{array}{l}\text { 110/H9/.8: Thin-Film Permanent } \\
\text { Magnets: } \\
\text { SALON 1-3 }\end{array}$ & & & \\
\hline $\begin{array}{l}\text { J10/KG/AA5: Spin Dynamics and } \\
\text { Transport SALON 1-3 } \\
\text { J11: Nanomagnet Arrays } \\
\text { Assemblies }\end{array}$ & $\begin{array}{l}\text { J12: Spin-Depend. Tunneling I } \\
\text { J13: Spin Filtering } \\
\text { SALON } 1-3\end{array}$ & & $\begin{array}{l}\text { J14: Spin-Depend. Tunneling II } \\
\text { J15: Spin-Valve Devices } \\
\text { SALON 1-3 }\end{array}$ & & & & \\
\hline \multicolumn{8}{|l|}{$\begin{array}{l}\text { Assemblies } \\
\text { K6/J10/AA5: Spin Dynamics and } \\
\text { Transport } \\
\text { SALON 1-3 }\end{array}$} \\
\hline $\begin{array}{l}\text { L4: Thin-Film Growth-Surface } \\
\text { Roughness, Texture }\end{array}$ & $\begin{array}{l}\text { L5: Film Microstructure and } \\
\text { Stress }\end{array}$ & $\begin{array}{l}\text { L6: Posters } \\
\text { (M) }\end{array}$ & $\begin{array}{l}\text { L7/19: Nanocrystalline Magnetic } \\
\text { Thin Fins } \\
\text { SALON } 4 / 5\end{array}$ & $\begin{array}{l}\text { L8/H9/110: Thin-Film Permanent } \\
\text { Magnets } \\
\text { SALON 1-3 }\end{array}$ & & & \\
\hline $\begin{array}{l}\text { M3: Electromigration } \\
\text { Mechanisms and Modeling }\end{array}$ & $\begin{array}{l}\text { M4: Electromigration Measure- } \\
\text { ments in Advanced Inter- } \\
\text { connects }\end{array}$ & $\begin{array}{l}\text { M5-M7: } \\
\text { Posters } \\
\text { (M) }\end{array}$ & $\begin{array}{l}\text { M8: Mechanical Behavior of } \\
\text { Back-End Materials }\end{array}$ & M9: Adhesion and Fracture & & & \\
\hline N6: Aluminum & $\begin{array}{l}\text { N7/O4: Low-k/Advanced } \\
\text { Interconnect }\end{array}$ & & & & & & \\
\hline O3: Low-k Integration & $\begin{array}{l}\text { O4/N7: Low-k/Advanced } \\
\text { Interconnect } \\
\text { GOLDEN GATE B2 }\end{array}$ & $\begin{array}{l}\text { O5: Posters } \\
\text { (M) }\end{array}$ & $\begin{array}{l}\text { O6: Low-k Film Property/ } \\
\text { Integration }\end{array}$ & & & & \\
\hline P5: CMP Modeling and Fluid Flow & $\begin{array}{l}\text { P6: Particle Adhesion and Post- } \\
\text { Polish Cleaning }\end{array}$ & & & 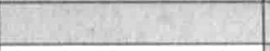 & & & \\
\hline $\begin{array}{l}\text { Q1: Surface Conditioning and } \\
\text { Analysis }\end{array}$ & $\begin{array}{l}\text { Q2: Organic and Particulate } \\
\text { Removal Processes }\end{array}$ & & $\begin{array}{l}\text { Q3: Pre-Gate and Chemical- } \\
\text { Mechanical Polishing } \\
\text { Applications }\end{array}$ & $\begin{array}{l}\text { O4: Novel Processes and } \\
\text { Applications }\end{array}$ & & & \\
\hline $\begin{array}{l}\text { R7: Advances in Ultrathin } \\
\text { Oxides and Oxynitrides II } \\
\text { R8: Silicon Nitinde II } \\
\text { NOB HILL C/D }\end{array}$ & $\begin{array}{l}\text { R9: High-k Altemate Gate } \\
\text { Dielectrics I } \\
\text { NOB HILL. C/D }\end{array}$ & $\begin{array}{l}\text { R10: Posters } \\
\text { (M) }\end{array}$ & $\begin{array}{l}\text { R11: Characterization of Gate } \\
\text { Dielectrics } \\
\text { NOB HILL C/D }\end{array}$ & $\begin{array}{l}\text { R12: High-k Alternate Gate } \\
\text { Dielectrics II } \\
\text { R13: Integrated Processing } \\
\text { NOB HILL CID }\end{array}$ & & & \\
\hline $\begin{array}{l}\text { S4: Physics of Dopants and } \\
\text { Defects }\end{array}$ & S5: Modeling & $x^{1}+\frac{1}{4}$ & S6: TED & $\begin{array}{l}\text { S7: Surface and Interface } \\
\text { Effects } \\
\text { S8: } 2 \text { D Profiling }\end{array}$ & & S9: SiGe and Nitrogen & \\
\hline T6: Water Bonding II and SOS & $\begin{array}{l}\text { T7; Compliant Substrates and } \\
\text { sos }\end{array}$ & & & & & & \\
\hline $\begin{array}{l}\text { U4: In Situ X-Ray. TEM, STMAFM } \\
\text { Characterization and Process- } \\
\text { ing Control }\end{array}$ & $\begin{array}{l}\text { U5; In Situ Emission and Optical } \\
\text { Spectroscopies, and Other } \\
\text { Characterization Techniques }\end{array}$ & 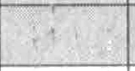 & & & & & \\
\hline V5: Growth Mechanisms & $\begin{array}{l}\text { V6/BB5: Epitaxial Multicomponent } \\
\text { Oxide Film Growth } \\
\text { SALON } 7\end{array}$ & \begin{tabular}{|l|} 
V7: Posters \\
(M)
\end{tabular} & V8: Novel Epitaxy & $\begin{array}{l}\text { V9/W11: Structural Charac- } \\
\text { terization \& Growth II } \\
\text { GOLDEN GATE C2 } \\
\end{array}$ & & & \\
\hline $\begin{array}{l}\text { W6: Self-Assembled, Embedded } \\
\text { SK Dols }\end{array}$ & $\begin{array}{l}\text { W7: Phonons in Dots } \\
\text { W8: Transport, Coulomb, } \\
\text { Biocade and Metallic Dots }\end{array}$ & $\begin{array}{l}\text { W9: Posters } \\
\text { (M) }\end{array}$ & $\begin{array}{l}\text { W10: Structural Characterization } \\
\text { and Growth I }\end{array}$ & $\begin{array}{l}\text { W11/V9: Structural Charac- } \\
\text { terization \& Growth it }\end{array}$ & & & \\
\hline$x$ & $x_{3}$ & $\sqrt{302908}$ & & $x_{4}$ & & & \\
\hline $\begin{array}{l}\text { Y6: GaN Growh and } \\
\text { Characterization }\end{array}$ & $\begin{array}{l}\text { Y7: III-N Growth and } \\
\text { Characterization }\end{array}$ & $\begin{array}{l}\text { Y8. Panel } \\
\text { Discussion }\end{array}$ & $\begin{array}{l}\text { Y9: GaN Devices and } \\
\text { Processing }\end{array}$ & & & & \\
\hline $\begin{array}{l}\text { 25: Electron Devices and } \\
\text { Processing I }\end{array}$ & $\begin{array}{l}\text { 26: Electron Devices and } \\
\text { Processing II }\end{array}$ & & & & & & \\
\hline \multicolumn{8}{|l|}{$\begin{array}{l}\text { AA5/J9/K6: Spin Dynamics and } \\
\text { Transport } \\
\text { SALON 1-3 }\end{array}$} \\
\hline $\begin{array}{l}\text { 8B4: Multicomponent Oxide } \\
\text { Devices I }\end{array}$ & $\begin{array}{l}\text { B85/V6: Epitaxial Multicomponent } \\
\text { Oxide Film Growth }\end{array}$ & \begin{tabular}{|l|} 
BB6, B87: \\
Posters (M) \\
\end{tabular} & $\begin{array}{l}\text { BB8: Multicomponent Oxide } \\
\text { Devices II }\end{array}$ & $\begin{array}{l}\text { B89: Properties, Characteriza- } \\
\text { tion, and Modeling }\end{array}$ & & & \\
\hline CC6: Anodes and NiMH & CC7: Supercapacitors & & $\begin{array}{l}\text { CC8: Solid Oxide Conductors } \\
\text { and SOFCS }\end{array}$ & C9: Solid Oxide Fuel Cells & & & \\
\hline $\begin{array}{l}\text { DD6: Meso-, Micro, and Macro. } \\
\text { Porous Hybrids }\end{array}$ & $\begin{array}{l}\text { DD7: Hybrid Electrochemistry, } \\
\text { Photochemistry, Passivation, } \\
\text { and Sensing }\end{array}$ & $\begin{array}{l}\text { DD8, DD9: } \\
\text { Posters } \\
\text { (M) }\end{array}$ & $\begin{array}{l}\text { DD10: Biomedical Applications of } \\
\text { Hybrid Materials } \\
\text { Db11: Electronic Properties and } \\
\text { Applications }\end{array}$ & $\begin{array}{l}\text { DD12: Mechanical Properties } \\
\text { and Applications }\end{array}$ & & $\begin{array}{l}\text { DD 13: Optical Properties and } \\
\text { Applications }\end{array}$ & \\
\hline \begin{tabular}{|l} 
FF1: Biodegradable Polymers \\
\end{tabular} & $\begin{array}{l}\text { FF2: In Vitro and } \ln \text { Vivo } \\
\text { Evaluations of Biomaterials }\end{array}$ & $\begin{array}{l}\text { FF3: Posters } \\
\text { (M) }\end{array}$ & e. & & & & \\
\hline GG3: Biomembrahes & $\begin{array}{l}\text { GG4: Hybrid Membranes } \\
\text { GGS: Bilayer/Multilayer } \\
\text { Membranes and Sensors }\end{array}$ & $\begin{array}{l}\text { GG6: Posters } \\
\text { (M) }\end{array}$ & & & & & \\
\hline HH5: Paper, Fiber, Polymers & & $\begin{array}{l}\text { HH6: Posters } \\
\text { (M) }\end{array}$ & & & & & \\
\hline
\end{tabular}




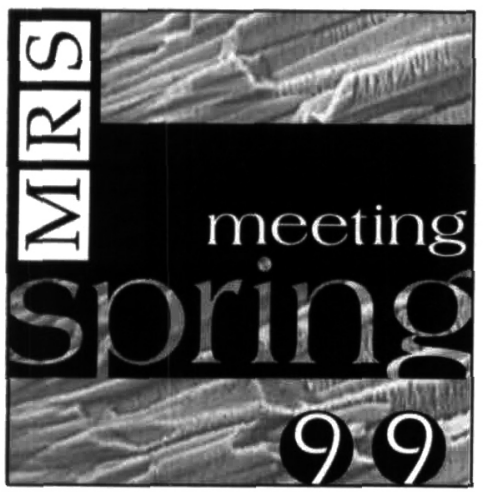

Forthcoming from MRS at special pre-meeting prices...

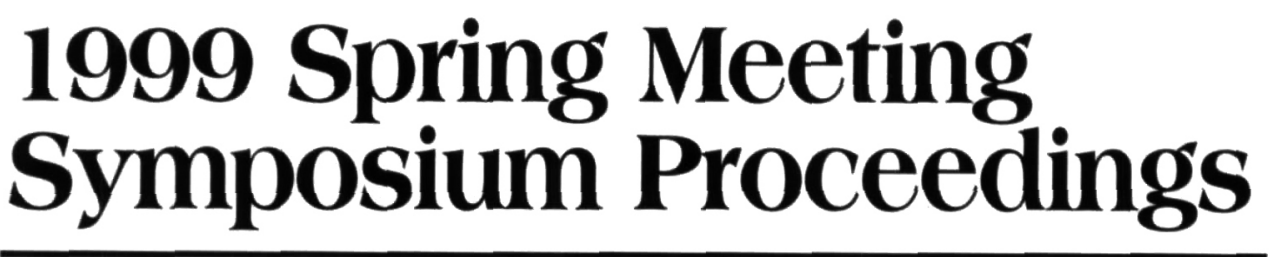

Place your order today for proceedings of the 1999 MRS Spring Meeting in San Francisco and SAVE!

Special pre-meeting prices (prices on left) effective until April 30, 1999. After April 30, 1999, pay the higher prices on the right.

A: Amorphous and Heterogeneous Silicon Thin Films: Fundamentals to Devices-1999

Editors: H.M. Branz, R.W. Collins, H. Okamoto,

S. Guha, R. Schropp

ISBN: 1-55899-464-5

$\begin{array}{lll}\$ 60.00 & \$ 65.00 & \text { MRS Member } \\ \$ 70.00 & \$ 77.00 & \text { U.S. List } \\ \$ 77.00 & \$ 85.00 & \text { Non-U.S. }\end{array}$

B: Flat-Panel Displays and SensorsPrinciples, Materials and Processes Editors: F.R. Libsch, B. Chalamala, R. Friend, T. Jackson, H. Ohshima

ISBN: 1-55899-465-3

$\begin{array}{lll}\$ 56.00 & \$ 62.00 & \text { MRS Member } \\ \$ 66.00 & \$ 73.00 & \text { U.S. List } \\ \$ 73.00 & \$ 80.00 & \text { Non-U.S. }\end{array}$

D: Liquid Crystal Materials and Devices Editors: T.J. Bunning, S.H. Chen, L.C. Chien, S-C.A. Lien, T. Kailyama, N. Koide

ISBN: 1-55899-466-1 Code: 559-B

$\begin{array}{lll}\$ 53.00 & \$ 58.00 & \text { MRS Member } \\ \$ 62.00 & \$ 68.00 & \text { U.S. List } \\ \$ 68.00 & \$ 75.00 & \text { Non-U.S. }\end{array}$

$\$ 68.00 \quad \$ 75.00 \quad$ Non-U.S.

E: Luminescent Materials

Editors: J. McKittrick, A. Kitai, K. Mishra, C. Ronda, B. DiBartolo

ISBN: 1-55899-467-X

$\begin{array}{lll}\$ 56.00 & \$ 62.00 & \text { Code: } 560-B \\ \$ 66.00 & \$ 73.00 & \text { MRS Member } \\ \$ 73.00 & \$ 80.00 & \text { U.S. List } \\ & \text { Non-U.S. }\end{array}$

$\$ 80.00 \quad$ Non-U.S

F: Organic Nonlinear Optical Materials and Devices

Editors: B. Kippelen, H. Lackritz, R. Claus

ISBN: 1-55899-468-8 Code: 561-B

$\begin{array}{lll}\$ 55.00 & \$ 61.00 & \text { MRS Member } \\ \$ 65.00 & \$ 72.00 & \text { U.S. List } \\ \$ 72.00 & \$ 79.00 & \text { Non-U.S. }\end{array}$

L: Polycrystalline Metal and Magnetic Thin Films

Editors: K.P. Rodbell, D. Laughlin, O. Thomas, B. Zhang

ISBN: 1-55899-469-6

$\$ 54.00$

$\$ 59.00$

Code: $562-B$

$\$ 63.00$

MRS Member

$\$ 69.00$

$\$ 69.00$

U.S. List

Non-U.S.

M: Materials Reliability in Microelectronics IX Editors: C.A. Volkert, A.H. Verbruggen, D. Brown

ISBN: 1-55899-470-X Code: 563-B

$\$ 55.00$

$\$ 61.00$

MRS Member

$\$ 65.00$

$\$ 72.00$

U.S. List

$\$ 72.00$
N: Advanced Interconnects and Contacts Editors: D.C. Edelstein, T. Kikkawa, M. Ozturk,

K-N. Tu, E. Weitzman

ISBN: 1-55899-471-8

$\begin{array}{lll}\text { BN: } 1-55899-471-8 & \text { Code: } 564-B \\ \$ 56.00 & \$ 62.00 & \text { MRS Member } \\ \$ 66.00 & \$ 73.00 & \text { U.S. List } \\ \$ 73.00 & \$ 80.00 & \text { Non-U.S. }\end{array}$

O: Low-Dielectric Constant Materials V Editors: J.P. Hummel, K. Endo, W.W. Lee, M.E. Mills, $S-Q$. Wang

$\begin{array}{ccc}\text { ISBN: } 1-55899-472-6 & \text { Code: } 565-B \\ \$ 54.00 & \$ 60.00 & \text { MRS Member } \\ \$ 64.00 & \$ 70.00 & \text { U.S. List } \\ \$ 70.00 & \$ 7.00 & \text { N. }\end{array}$

$\$ 70.00 \$ 7.00 \quad$ Non-U.S.

P: Chemical-Mechanical PolishingFundamentals and Challenges

Editors: S.V. Babu, S. Danyluk, M.I. Krishnan, M. Tsujimura

$\begin{array}{ccc}\text { ISBN: } 1-55899-473-4 & \text { Code: } 566-B \\ \$ 54.00 & \$ 60.00 & \text { MRS Member } \\ \$ 64.00 & \$ 70.00 & \text { U.S. List } \\ \$ 70.00 & \$ 7.00 & \text { Non-U.S. }\end{array}$

R: Ultrathin $\mathrm{SiO}_{2}$ and High-K Materials for ULSI Gate Dielectrics

Editors: H.R. Huff, M.L. Green, T. Hattori, G. Lucovsky, C.A. Richter

ISBN: 1-55899-474-2

$\begin{array}{lll}\$ 58.00 & \$ 64.00 & \text { MRS Member } \\ \$ 68.00 & \$ 75.00 & \text { U.S. List } \\ \$ 75.00 & \$ 82.00 & \text { Non-U.S. }\end{array}$

S: Silicon Front-End ProcessingPhysics and Technology of Dopant-Defect Interactions

Editors: H-J.L. Gossmann, T.E. Haynes, M.E. Law,

A.N. Larsen, S. Odanaka

ISBN: 1-55899-475-0

$\begin{array}{lll}\$ 54.00 & \$ 60.00 & \text { MRS Member } \\ \$ 64.00 & \$ 70.00 & \text { U.S. List } \\ \$ 70.00 & \$ 77.00 & \text { Non-U.S. }\end{array}$

U: In Situ Process Diagnostics and Modelling Editors: O. Auciello, A.R. Krauss, E.A. Irene, J.A. Schultz

$\begin{array}{ccc}\text { ISBN: } 1-55899-476-9 & \text { Code: } 569-B \\ \$ 54.00 & \$ 60.00 & \text { MRS Member } \\ \$ 64.00 & \$ 70.00 & \text { U.S. List } \\ \$ 70.00 & \$ 77.00 & \text { Non-U.S. }\end{array}$

V: Epitaxial Growth

Editors: T.P. Pearsall, A-L. Barabasi, F. Liu,

G.N. Maracas

ISBN: 1-55899-477-7

$\$ 56.00$

$\$ 66.00$

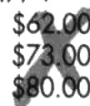
Code: $570-B$ MRS Member U.S. List Non-U.S.
W: Semiconductor Quantum Dots

Editors: H. Lee, S. Moss

ISBN: 1-55899-478-5

$\begin{array}{lll}\text { BN: } 1-55899-478-5 & \text { Code: } 571-\text { B } \\ \$ 57.00 & \$ 63.00 & \text { MRS Member } \\ \$ 67.00 & \$ 74.00 & \text { U.S. List } \\ \$ 74.00 & \$ 81.00 & \text { Non-U.S. }\end{array}$

Y: Wide-Bandgap Semiconductors for High-Power, High-Frequency and High-Temperature Applications-1999

Editors: S. Binari, A. Burk, M. Melloch, C. Nguyen ISBN: 1-55899-479-3 Code: 572-B

$\begin{array}{lll}\$ 57.00 & \$ 63.00 & \text { MRS Member } \\ \$ 67.00 & \$ 74.00 & \text { U.S. List } \\ \$ 74.00 & \$ 81.00 & \text { Non-U.S. }\end{array}$

Z: Compound Semiconductor Surface Passivation and Novel Device Processing Editors: H. Hasegawa, M. Hong, Z.H. Lu, S.J. Pearton ISBN: 1-55899-480-7 Code: 573-B

$\begin{array}{lll}\$ 54.00 & \$ 60.00 & \text { MRS Member } \\ \$ 64.00 & \$ 70.00 & \text { U.S. List } \\ \$ 70.00 & \$ 7.00 & \text { Non-U.S. }\end{array}$

BB: Multicomponent Oxide Films for Electronics

Editors: M.E. Hawley, D.H. Blank, C-B. Eom

S.K. Streiffer, D.G. Schlom

ISBN: 1-55899-481-5

$\$ 58.00 \quad \$ 64.00$

$\$ 68.00$

$\$ 64.00$

$\$ 75.00$

$\$ 82.00$

Code: $574-B$

MRS Member

U.S. List

Non-U.S.

CC: New Materials for Batteries and Fuel Cells Editors: D.H. Doughty, H-P. Brack, K. Naoi, L.F. Nazar ISBN: 1-55899-482-3

$\begin{array}{lll}\$ 56.00 & \$ 62.00 & \text { MRS Member } \\ \$ 66.00 & \$ 73.00 & \text { U.S. List } \\ \$ 73.00 & \$ 80.00 & \text { Non-U.S. }\end{array}$

DD: Organic/Inorganic Hybrid Materials II Editors: L.C. Klein, L. Francis, M.R. DeGuire,

J.E. Mark

$\begin{array}{ccc}\text { ISBN: } 1-55899-483-1 & \text { Code: } 576-B \\ \$ 57.00 & \$ 63.00 & \text { MRS Member } \\ \$ 67.00 & \$ 74.00 & \text { U.S. List } \\ \$ 74.00 & \$ 81.00 & \text { Non-U.S. }\end{array}$

\section{Order NOW and SAVE!}

These books are scheduled for publication by fall or early winter 1999.

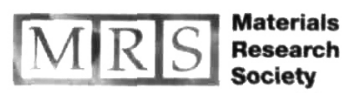

506 Keystone Drive, Warrendale, PA 15086-7573 U.S.A Telephone: 724-779-3003 • Fax: 724-779-8313

E-mail: info@mrs.org • mmw.mrs.org/publications/books/ 


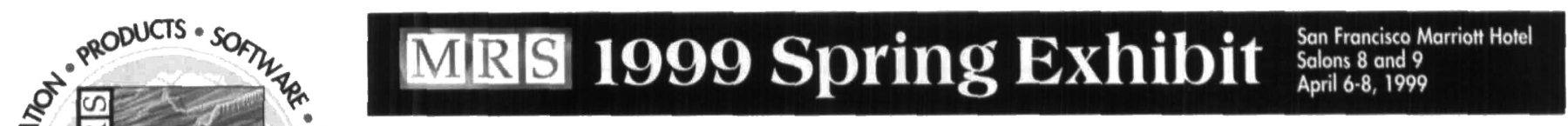

Exhibit Hours:

Tuesday, April 6

Wednesday, April 7

Thursday, April 8

Complimentary reception will be held in the exhibit hall on Tuesday evening from $5: 00 \mathrm{pm}$ to $6: 30 \mathrm{pm}$.
The MRS Exhibit, held in conjunction with the 1999 MRS Spring Meeting, will encomposs the full spectrum of equipment, instrumentation, products, softwore, publications and servicas for materials reseorch. As ahwoys, the exhibit will closely poralled the nature of the lochnical symposia, and the progrom has been arronged to allow meeting participants ample opportunity to visit the exhibit. MRS encourages antendees to visit the exhibit by scheduling coffee breaks, deli-syle lunches, and a meeting-wide reception in the exhibit hall.

\section{Partial List of 1999 Spring Exhibitors (os of January 22, 1999)}

A \& $N$ Corporation \#215

707 Southwest 19 th Avenue

Williston, FL 32696

Tel: $352-528-4100$

Fax: 352-528-3441

E-mail: info@ancorp.com

www.ancorp.com

A \& N Corporation has been a manufacturer of high-quality vacuum components for over 30 years. Our product line includes flanges and fittings in the following styles ISO-KF (QF), ISO-MF (LF), UHV (CF), ASA tri-seal, vacuum couplings, feed throughs, vacuum ball valves, and special fabrications New for 1999: right angle valves, in-line valves, gate valves, and vacuum line heaters/controllers.

\section{- ABB Extrel \#603}

575 Epsilon Drive

Pittsburgh, PA 15238-2838

Tel: 412-963-7530

Fax: 412-963-6578

E-mail: qms@extrel.com

www.abb.com/extrel

ABB Extrel has been manufacturing quadrupole mass spectrometers and systems since 1964. Our instruments' high sensitivity and resolution allow you to understand and control process chemistries that simple RGAs cannot. Our instruments are used for plasma, CVD and environmental monitoring, surface science studies, ion chemistry and high purity gas analysis.

\section{Acodemic Press \#120}

525 B Street, Suite 1900

San Diego, CA 92101

Tel: 619-699-6774

Toll Fee: 800-321-5068

Fax: 800-874-6418

E-mail: ap@acad.com

www.academicpress.com

Browse our new releases including

Handbook of Vacuum Science and Technology,

Reliability and Failure of Thin Films, Materials

Science of Thin Filnts, Quantum Semiconductor

Structures, Thin Films: PVD for

Microelectronics, Vol. 26, Theoretical and

Mathematical Models in Polymer Research,

Biorelated Polymers and Gels, Surface Activity

and Giant Molecules.

\section{Accurel Systems/Moterials Anabysis}

Group \#604

785 Luceme Drive

Sunnyvale, CA 94086

Tel: $408-737-3892$

Fax: 408-737-3916

E-mail: suzannef@accurel.com

www.accurel.com

Accurel Systems and Materials Analysis Group provide the most comprehensive range of surface, materials, and failure analysis services. Rely on Accurel as your one-stop analytical resource, and experience personalized, quality service when you need it. Capabilities include FIB, dual beam FIB, flip chart FIB, FE-SEM, CIVVA, TEM, EDX SIMS, AFM, FE-AES, ESCA, turnkey failure analysis, emission microscopy, e-beam probing, deprocessing, decapping, quick-turm packaging, real time $x-r a y, S A M$ and XRD.
A-VAC Industries \#317

$1845 \mathrm{~S}$. Lewis Street

Anaheim, CA 92805

Tel: 714-938-1300

Toll Free: 800-747-2821

Fax: 714-935-0899

E-mail: avac@earthlink.net

A-VAC Industries has specialized in high vacuum equipment sales, service and support since 1969. New and rebuilt helium leak detectors, mechanical pumps, cryo, turbo, diffusion pumps and more Professional rebuilding available for customer equipment and a huge parts accessories department to insure customer up-time. Call today for a free catalog.

\section{Advanced Materials Instruments \& Anatysis} \#108

7801 North Lamar Blvd., Suite C-63

Austin, TX 78752

Tel: 512-452-7862

Fax: 512-452-1972

E-mail: amia@amia.com

www.amia.com

A service laboratory providing $\mathrm{x}$-ray diffraction analysis on semiconductors, polymers and other advanced materials, special. izing in texture, stress, thin film measurements (reflectometry) and grain size. Also provides analysis using EBSB (Kikuchi patterns) for crystal orientation and mapping and phase identification. Manufacturers of custom $\mathrm{x}$-ray diffraction instruments.

\section{Advanced Research Systems \#101}

905 Harrison Street, Suite 109

Allentown, PA 18103

Tel: $610-439-8022$

Fax: 610-439-1184

E-mail: arscryo@aol.com

Advanced Research Systems offers a complete line of cryogenic systems for low temperature research. Products include Displex closed-cycle systems and Heli-tran flow cryostats for studies such as UHV, optical (UV, Vis, IR, $x$-ray) and resistivity. We'll be happy to work with you to design custom interfaces to meet your specific needs, and we also offer a full line of temperature controllers and sensors for all applications.

\section{- AIXTRON, Inc. \#211}

1670 Banclay Blvd

Buffalo Grove, IL 60089

Tel: 847-215-7335

Fax: 847-215-7341

ALXTRON is the leading manufacturer of

MOCVD and VPE equipment for the growth of all III-V, including ritrides, II-VI oxides and $\mathrm{SiC}$. Outstanding quality in manufacturing, reliability in safety, and highest uniformity results for epilayers. Systems include: AIX-200; AIX200/4 AIX-2400; the world's largest, the AIX-3000; and the new, automated $2600 \mathrm{G} 3$

\section{AJA Intemational, Inc. \#402}

P.O. Box 246

809 Country Way

N. Scituate, MA 02060

Tel: 781-545-7365

Fax: 781-545-4105

E-mail: topgun@ajaint.com

www.ajaint.com

Circular and rectangular magnetron

sputtering sources and targets, ATC R\&D sputtering systems, substrate heaters, RF and $D C$ power supplies, microwave powe supplies and components, microwave plas ma sources (downstream, surfaguide, SLAN, surfatron), diamond film equipment, electrostatic chucks and ESC power supplies.

\section{- Aldrich Chemical Company, Inc. \#407}

P.O. Box 355

Milwaukee, WI 53201

Tel: 414-298-7925

Fax: 414-298-7960

E-mail: crecatto@sial.com

www.sigma-aldrich.com

Aldrich produces and stocks a wide range of chemical products-including elemental forms, alloys, high purity inorganics, anhy drous metal halides, high purity gases and organometallics for CVD, ligands, ACS reagents, and analytical standards-used in scientific research, product development and analysis in the inorganic, organometallic, and materials science fields.

American Institute of Physics, Inc, \#123

500 Sunnyside Blvd.

Woodbury, NY 11797

Tel: 516-576-2484

Fax: 516-576-2374

E-mail: corr@aip.org

www.aip.org

Learn how your subscription to any AIP journal connects you to a powerful hyperlinked library of physical science data. Our Online Journal Service (OJS) now offers reference linking to INSPEC and SIIN databases, online document delivery, and the ability to download articles in a variety of formats.

\section{- Bede Scientific Incorporated \#515}

14 Inverness Drive East, Suite G-104

Englewood, CO 80112

Tel: $303-790-8647$

Fax: $303-790-8648$

E-mail: info@bede.com

www.bede.com

Bede Scientific is a world leader in materials characterization, established for many years in high resolution $x$-ray diffraction and scattering techniques, langely in the semiconductor industry. Bede developed the world's first quality control diffractometer, while recent advances include combined XRD and photoluminescence mapping. The range of research instruments includes the highest performance diffractometers available on the market. Bede's analytical software is accepted as an industry standard and has recently been extended by automatic parameter fitting programs based upon powerful generic algorithms.

\section{Bioanalytical Systems, Inc. (BAS) \#112}

2701 Kent Avenue

West Lafayette, IN 47906-1382

Toll Free: 800-845-4246

Fax: 765-497-1102

E-mail: echem@bioanalytical.com wwwbioanalytical com

BAS manufactures and distributes a comprehensive line of electrochemical equipment, including potentiostats, galvanostat impedance analyzers, and electrodes. BAS will be exhibiting the BAS $100 \mathrm{~B} / \mathrm{W}$

Electrochemical Workstation, the RDE-1 Rotating Disk Electrode, the BAS-Zahner

IM6 Impedance Analyzer, and software for simulation of cyclic voltammetry (DigiSim (R)).

- Blake Industries, Inc. \#200

660 Jerusalem Road

Scotch Plains, NJ 07076

Tel: 908-233-7240

Fax: 908-233-1354

E-mail: blake4xray worldnet.att.net Blake Industries will be exhibiting Huber rotary tables, translation stages, goniometer heads, $X-Y$ slits for synchrotron and rotating anode experiments. Blake monochromators, thin-film cameras, and Laue equipment will also be displayed.

\section{BOC Edwards \#507}

301 Ballardvale Street

Wilmington, MA 0188?

Tel: $800-848-9800$

Fax: 978-658-7969

www bocedwands com

BOC Edwards offers a complete line of high quality vacuum components and systems. Products include drypumps, rotary vane pumps, turbomolecular pumps, vacuum instrumentation, leak detectors, thin film coaters and a range of vacuum accessories. Our Vacuum Training Center offers courses ranging from general vacuum practices to specific product reviews. Our Applications Engineering team offers innovative solutions to optimize your vacuum system and 24-hour product support is available.

\section{Burleigh Instruments, Inc. \#502}

P.O. Box E

7647 Main Street, Burleigh Park

Fishers, NY 14453-0755

Tel: 716-924-9355

Fax: 716-924-9072

E-mail: info@burleigh.com

www.burleigh.com

Burleigh Instruments designs and manufac- 


\section{CHA Industries \#617}

4201 Business Center Drive

Fremont, CA 94538-6357

Tel: $510-683-8554$

Fax: 510-683-3848

www.chaindustries.com

CHA Industries is vertically integrated, with over 50 years of experience as a manufacturer and OEM supplier of standard and custom high-vacuum systems.

Manufacturers of semiconductor, XTAL, LEDs, optics, automotive, and aerospace devices use various versions of CHA's sputtering, evaporation, and ion beam systems. CHA manufactures roll coaters, box coaters, clean-room compatible $R \& D$, and production systems (and components) for medium-and high-vacuum applications.

- Chemat Technology, Inc. \#214, 216

9036 Winnetka Avenue

Northridge, CA 91324

Tel: $818-727-9786$

Fax: 818-727-9477

E-mail: chemat@aol.com

Chemat Technology, Inc. is the leading resource for total sol-gel processing. With centers woridwide, services offened include research and development, manufacture and distribution of metalorganic precursor for CVD and sol-gel, and the design and construction of thin film coating equipment. Standard and customized equipment range from low-cost spincoaters to sophisticated dipcoating systerns. Chemicals are available in research and bulk quantities. Chemat's distinguishing feature is the ability to satisfy unique individual requirements.

- Commonweath Scientific Corporation 1600

500 Pendleton Street

Alexandria, VA 2231

Tel: 703-548-0800

Fax: 703-548-7405

E-mail: cscionbeam.com

Commonwealth Scientific Corporation (CSC) is the leader in ion beam technology, manufacturing a complete line of ion beam sources and systems for surface modification processes including nitriding, dry etching and thin film deposition. R\&D and production batch, load lock and cluster tool system configurations to perform ion beam deposition and etching for MR, GMR and DLC applications.

\section{Coming Engineering Laboratory Services (CELS) \#109 \\ Corning, NY 1483 \\ Tel: 607-974-6154 \\ Fax: 607-974-6522 \\ E-mail: cels@corning.com \\ www.cels.corning.com \\ CELS is a commercial laboratory offering quality testing in areas of chemical analysis, surface analysis, physical property testing, defect analysis, mechanical property testing electrical property testing, particle characteri- zation, mixing and melting studies, refracto- ry property testing and thermal analysis. Areas of expertise include glasses, ceramics, refractories, raw materials, organics, inorgan ics, polymers, surfaces, metals and more.}

- Cree Reseorch, Inc. \#518

4600 Silicon Drive

Durham, NC 27703

Tel: $919-313-5300$

Fax: 919-313-5451

www.cree.com

Cree manufactures $4 \mathrm{H}-\mathrm{SiC}$ and $6 \mathrm{H}-\mathrm{SiC}$ substrates and epitaxy, semi-insulating $4 \mathrm{H}-\mathrm{SiC}$ substrates, high intensity blue LEDs and SiC UV photodiodes. SiC is an exceptional material for nitride deposition and the fabrication of semiconductor devices for high temperature, high power, high frequency power and optoelectronic applications. Custom device/design services available.
Cryomech, Inc. \#405

113 Falso Drive

Syracuse, NY 13211

Tel: 315-455-2555

Fax: 315-455-2544

E-mail: specs@cryomech.com

www.cryomech.com

NEW FROM CRYOMECH: A 4.2 K Pulse

Tube Cryorefrigerator with 0.500 watts at

$4.2 \mathrm{~K}$. Cryomech also manufactures standard laboratory cryostats, Gifford-McMahon cycle cryorefrigerators and liquid nitrogen plants. Customized cryostats for the unique experiment are available.

\section{Crystallographica \#217}

3 Blenheim Office Park

Lower Road, Long Hanborough

Oxfordshire OX8 8L N

United Kingdom

Tel: 44-1993-883488

Fax: 44-1993-883988

E-mail: info@oxfordcryosystems.co.uk www.crystallographica.co uk

Crystallographica is the software department of Oxford Cryosystems and specializes in software for powder diffraction. Products include Crystallographica, the crystallographic software tool kit for Microsoft Windows, and has just been extended to include Crystallgroaphica Seanch-Match (CSM). CSM is a 32-bit search match program for use with the International Centre for Diffraction Data's Powder Diffraction File. The Crystallographica team is continually developing new ideas for both existing and new products to provide the best qualty crystallographic software products.

\section{DCA Instruments, Inc. \#519}

1 Hilltop Lane

White Plains, NY 10607-1709

Tel: $914-421-1969$

Fax: 914-946-6766

E-mail: dcausa@att.net

www.dca.co.uk

DCA Instruments specializes in the design and manufacture of high-quality UHV deposition systems, offering standard systems for the following deposition techniques: III-V, II-VI, CMT-MBE, metal MBE, UHV sputtering, UHV laser ablation, and UHV CVD. DCA Instruments also offers a wide range of MBE components which are retrofittable to the majority of existing systems. Components include effusion cells, soft-action magnetically driven linear shutters, a 'zero-wobble' substrate manipulator and a self-regulating mercury source.

- Denton Vacuum, LC \#314

1259 North Church Street

Moorestown, NJ 08057

Tel: $609-439-9100$

Fax: 609-439-9111

E-mail: info@dentonvacuum.com

www.dentonvacuum.com

Denton Vacuum, LLC is a leading manufacturer of vacuum deposition systems. The Integrity Series of evaporators features state-of-the-art technology for research or production. The Discovery ${ }^{18}$ Series of sputtering systems are ideal for research or small production requirements. Denton also manufactures an extensive line of small deposition systems suitable for microscope sample preparation.

- Digital Instruments, Inc. \#511

112 Robin Hill Road

Santa Barbara, CA 93117

Tel: $805-967-1400$

Fax: 805-967-7717

E-mail: info@di.com

www.di.com

Digital Instruments, the world leader in scanning probe microscopy (SPM), manufactures a complete line of NanoScope ${ }^{\otimes}$ SPMs, including the MultiMode ${ }^{\mathrm{TM}}$ Atomic Force Microscope (AFM), the world's highest resolution SPM and the Dimension
Series ${ }^{\mathrm{TM}}$ SPMs, the world's best selling SPMs, offering the complete range of AFM techniques for small or large samples for the full range of materials science applications, including metals, films, ceramics, optics, plastics/polymers, paints and coatings, data storage devices, semiconductors and many others. We'll also be demonstrat ing our Phase Imaging Technique, a fast and easy method of differentiating region of differing composition, friction, adhesion viscoelasticity, etc. on sample surfaces.

\section{Duniway Stockroom Corp. \#613}

1305 Space Park Way

Mountain View, CA 94043-1336

Tel: $650-969-8811$

Fax: 650-965-0764

E-mail: info@duniway.com

www.duniway.com

New replacement parts for ion pumps, leak

detectors, and vacuum systems; including

12-point bolts, copper gaskets, TC gauges,

ion gauges, oil for diffusion pumps, etc.

Surplus vacuum equipment for sale, rebuil to original performance. Free catalog, including prices, shows new and surplus equipment.

\section{Eagle-Picher Technologies, LC \#404}

Electro-Optic Materials

P.O. Box 737

737 Highway 69A

Quapaw, OK 74363

Tel: $918-673-1650$

Fax: 918-673-2121

E-mail: epi-eom@mmind.net

The "EOM" Department of Eagle-Picher Industries, Inc. is a manufacturer of ultra high purity gallium metal, gallium trichloride, gallium sesquioxide, germanium tetrachloride, germanium dioxide, intrinsic germanium metal, germanium substrates, germanium, and silicon infrared optical materials. Eagle-Picher also purchases scrap gallium and germanium, in many forms, for recycling.

\section{- EDAX Inc. \#303}

1 McKee Drive

Mahwah, NJ 07430

Tel: 201-529-4880

Fax: 201-529-3156

E-mail: info@edax.com

www.edax.com

EDAX Inc. has perfected the way in $x$-ray chemical analysis of micro and bulk sample by energy dispersive microanalysis, $x$-ray spectrometry, detector technology electronics and applications. Our staff is ready to guide you in your choice of instrumentation you may need in any situation. EDAX will be presenting the Phoenix and the Falcon.

\section{Electro Optical Industries, Inc. \#207}

\section{Ward Drive}

Santa Barbara, CA 93111

Tel: 805-964-6701

Fax: 805-967-8590

E-mail: eoi@eoi-furnace.com

www.eoi-furnace.com

Electro Optical Industries manufactures low and high temperature atmosphere and high vacuum specialty furnaces. Applications include debinding, soldering, glass-treating, hot-pressing, vacuum and hydrogen brazing active metal brazing, ceramic sintering metallizing and cofiring and surface treatment.

Elsevier Science Inc. \#122, 124

655 Avenue of the Americas

New York, NY 10010-5107

Tel: $212-633-3766$

Fax: 212-633-3764

E-mail: usinfo-gelsevier.com

www.elsevier.com

Elsevier Science (imprints North-Holland,

Elsevier and Pergamon) will exhibit a wide range of materials science and related solid

state physics publications. Products on display include:

- Polymer Direct, the online version of the journal Polymer. Current awareness services including, Polymer Contents Online and Composites Online

- New journals including Journal of Inorganic Materials, Materials Science $\mathcal{E}$ Semiconductor Processing and Crystal Engineering

- Journals, such as Materiols Letters, Acto Materiala, Scripta Materiala, Materials Research Bulletin, Nanostructured Materials, Surface Science, Computational Materials Science

- Recently published books: CALPHADCalculation of Phase Diagrams: A Comprehensive Guide; Long Wave Polar Modes in Semiconductor Heterostructures; Properties of Polymers.

- The magazine and newsletterMaterials Today

\section{- EMCORE Corporation \#520}


Esdak Inc. \#106

7011 Anjou Creek Circle

San Jose, CA 95120

Tel: 408-997-6777

Fax: 408-997-7496

E-mail: esdak@msn.com

www.esdak.com

Esdak provides applications, service and sales support specifically to the metrology aspects of the high technology industry. The manufacturers' products represented are ellipsometers, spectroscopic systems, microwave $P C D$, four-point probes, $C V$ and upgrades to existing Esca and Auger equipment. We also supply used metrology equipment.

\section{- ESM Soffware \#III}

\section{Wade Court}

Hamilton, $\mathrm{OH} 45013$

Tel: $513-738-4773$

Fax: 216-274-902

E-mail: info@esm-software.com

www.esm-software.com

ESM Software will demonstrate its materials science software collection including the new version of the TAPP materials properties database, an updated NIST Crystal Data collection, new molecular modeling products SOLID and $\mathbf{k S a n}$, and updates of software for crystallography, thermochemistry, phase diagrams, kinetics, thin films, and materials properties.

\section{ESPI $\# 219$}

1050 Benson Way

Ashland, OR 97520

Tel: 800-638-2581

Fax: 541-488-8313

E-mail: sales@espi-metals.com

ESPI has been providing everything from Aluminum thru Zirconium to domestic and international manufacturing firms, the scientific community and educational institutions. You can depend on ESPI to supply you with a wide variety of high-purity metals, chemicals, rare earths, alloys and compounds, single crystals, sputtering targets, evaporation materials, precious metals, and other research materials.

\section{- Charles Evans \& Associates \#623,625}

301 Chesapeake Drive

Redwood City, CA 94063

Tel: $650-369-4567$

Fax: 650-369-7921

E-mail: info@cea.com

www.cea.com

Charles Evans \& Associates is an ISO 9002 certified commercial analytical laboratory providing surface and microanalytical services. We are committed to continuous improvement in the quality of our analysis procedures and measurements through ou Quality Assurance Program. Capabilities at our Redwood City site include SIMS (magnetic sector, quadrupole analyzer and timeof-flight mass spectrometers), XPS/ESCA RBS, HFS, PIXE, NRA, FTIR and TXRF. Our Sunnyvale site is equipped with electron beam and imaging techniques such as AES, SEM/EDS, SEM, FE-SEM, In-Lens FE-SEM and AFM/SPM.

FEl Beam Technology Division \#323

7425 NW Evergreen Parkway

Hillsboro, OR 97124

Tel: $503-844-2520$

Fax: 503-640-7509

E-mail: beamtech@feico.com

www,feibeamtech.com

FEI Beam Technology Division manufactures

and sells nanometer resolution, UHV-compatible, liquid metal ion and Schottky field emission electron focusing columns for

microscopy, Auger, SIMS, micromaching, and other surface science and semiconductor applications. Other products include $\mathrm{LaB}_{6}$ and $\mathrm{CeB}_{6}$ thermal emission electron cathodes.
- FEl Company/Philips Electron Optics $\$ 400$ 7451 NW Evergreen Parkway

Hillsboro, OR 97124

Tel: $503-640-7500$

Fax: 503-640-7509

E-mail: semisales@feico.com www.feic.com

FEI offers the broadest range of charged particle optics solutions available from single source: Philips SEMs, TEMs and ESEMs, and FEI FIB, Wafer SEM and DualBeam FIB/SEM workstations. Our solutions serve a broad range of materials research applications: from general materials analysis with SEM, to high magnification imaging and analysis of dynamic, hydrated or heated specimens using ESEM; from high resolution, atomic-level TEM evaluation of structures and interfaces, to FIB-based failune analysis and defect classification on integrated circuits. All FEI instruments are designed for easy integration of additional analytical hardware, and operate under Windows NT 4.0 making them familiar and easy-to-use for all skill levels.

\section{- Fischione Instrument \#408,410}

9003 Corporate Circle

Export, PA 15632

Tel: 724-325-5444

Fax: 724-325-5443

E-mail: info@fischione.com

www. fischione.com

Fischione Instruments produces a full line of TEM specimen preparation instrument including the disk punch, ultrasonic disk cutter, cross-section prep kit, dimpling grinder, twin-jet electropolisher and ion mill. Also featured are a series of plasma cleaners for the simultaneous removal of organic contamination from TEM and SEM specimens and specimen holders. Additional products are TEM specimen holders, including a single-tilt/rotate holder, a double-tilt analytical holder and a cryotransfer system, as well as imaging and analysis systems.

- Fujikin of America, Inc. \$100

2028 E. Ben White Blvd.

Suite 320

Austin, TX 7874]

Tel: 512-912-9095

Fax: 512-912-8095

E-mail: sales@fujikin.com www.fujikin.com

Fujikin is the world's leading manufacturer of valves and fittings for UHP applications. With surface finishes as low as $2 \mu$ in. $R a$ very low internal volume and extremely low particle generation, our conventional and surface mount products can take you to $300 \mathrm{~mm}, 180 \mathrm{~nm}$ and beyond. Contact us about our UHP steam generator and other culting-edge products.

\section{Gotan, Inc. \#315}

5933 Coronado Lane

Pleasanton, CA 94588

Tel: $925-463-0200$

Fax: 925-463-0204

E-mail: info@gatan.com

www.gatan.com

Gatan, Inc. is the world's leading manufacturer of instrumentation and software used to enthance and extend the operation and performance of electronmicroscopes. Gatan's products, which are fully compatible with all brandselectron microscopes, cover the entire range of the analytical process from specimen preparation and manipulation to imaging and analysis. Our customer base spans the complete spectrum of end-users of analytical instrumentation typically found in industrial, governmental, and academic laboratories. The applications addressed by these scientists and researchers include metallurgy, semicon ductors, electronics, biological science, new materials research and biotechnology. The Gatan brand name is very recognized and respected throughout the worldwide scientific community and has been synonymous with high quality products and the industry's leading technology.

\section{- Gelest lnc. \#321}

612 William Leigh Drive

Tullytown, PA 19007-6308

Tel: 215-547-1015

Fax: 215-547-2484

E-mail: info@gelest.com

www.gelest.com

Gelest is a manufacturer of metal-organics including silanes, silicones, germanium and tin compounds. Gelest also manufactures a broad range of metal alkoxides and metal diketonates. Products are used in microelectronic and optical coatings, sol-gel ceramics, composites and polymer synthesis.

\section{GFS Chemicals, Inc. \#104}

\section{PO. Box 245}

Powell, $\mathrm{OH} 43065-0245$

Tel: 800-858-9682

Fax: 740-881-5501

E-mail: gfschem@gfschemicals.com www gfschemicals com

Custom capabilities in both organic and inorganic specialties reflect the value GFS Chemicals offers to materials scientists.

Organics include cholesteryl testers for liquid crystals; various phenylethynyl aromatics for luminescent materials; other acetylenic intermediates for coatings and sensors. Inorganics include high purity salts as precursors to optical materials; lithium perchlorate and other salts and electrolyte blends for battery research; anhydrous rare earth halides and other specialty dry salts for composite R\&D; metal complexes of phenanthrolines and other aromatics. Build your research program on our 70 years of experience manufacturing custom and specialty materials.

- Goodfellow Corporation \#611

800 Lancaster Avenue

Berwyn, PA 19312-1780

Toll Free: $800-821-2870$

Fax: 800-283-2020

E-mail: info@goodfellow.com

www.goodfellow.com

Goodfellow supplies small quantities of metals and materials for research and development. Our capabilities range from one off prototypes to small production quantities.

\section{- High}

\section{P.O. Box 99}

$3800 \mathrm{AB}$ Amersfoort

The Netherlands

Tel: $31-33-4619741$

Fax: 31-33-461529

E-mail: info@highvolteng.com

www.highvolteng.com

High Voltage Engineering an engineering oriented company, designs, manufactures, sells and markets custom-made, high-tech capital equipment for the world market. Specializing in the development and manufacture of ion beam technology-based equipment, High Voltage Engineering is the largest and most diverse manufacturer of particle accelerator systems for the scientifc, educational and industrial research communities. Major product lines include: Ion Accelerator Systems, Research Ion

Implanters, Systems for Ion Beam Analysis, and various components such as HV power supplies, electron and ion accelerator tubes, ion sources, beamline components, beam monitoring equipment, etc.

- Huntington Mechanical Laboratories, Inc. $\# 60$

1040 L'Avenida Street

Mountain View, CA 94043-1422

Tel: 651-964-3323

Fax: 650-964-6153

E-mail: vacman@huntvac.com

www.huntvac.com

The industry's largest selection of vacuum valves, flanges, fittings, and feed throughs is available when you need it at Huntington. Also available are a wide assortment of roughing components including flexible hoses, traps, thermocouple and ionization gauge tubes, sorption and jet roughing pumps. Standard, custom, or modified $\mathrm{UHV}$ positioning and motion devices can be provided to meet your special needs. Stainless steel custom chambers, tees, and crosses are supported by a quarter of a century of experience in vacuum chamber design and fabrication at Huntington.

Hysitron, Inc. \#417

5251 West 73nd Street

Edina, MN 55439

Tel: $612-835-6366$

Fax; 612-835-6166

E-mail: hysitron@hysitron.com

www.hysitron.com 
expanding market. Standard product lines include multi-pin, coaxial, thermocouple and power feedthroughs; also, breaks, viewports and custorn design products.

\section{- Ion Tech, Ine. \#414,416}

2330 East Prospect

Fort Collins, CO 80525

Tel: 970-221-1807

Fax: 970-493-1439

E-mail: info@iontechinc.com

www.iontechinc.com

Ion Tech, Inc. is the industry leader in the design and manufacture of ion beam systems and sources to meet any research or production requirement. Our product line features thin film deposition and etch systems, DC and filamentless $R F$ ion beam sournes in both linear and round configurations, cylindrical magnetrons and power supplies.

\section{lonwerks \#105}

2472 Bolsover, Suite 255

Houston, TX 77005

Tel: 713-522-9880

Fax: 713-522-6735

E-mail: ionwerks@aol.com

wwwitionwerks.com

lonwerks is a provider of instrumentation and electronics for time-of-flight mass spectroscopy. Featured products are R\&ED 100 awand-winning Reflectron for SIMS/mass spectroscopy of recoiled ions and other custom-designed TOF ion scattering systems. Additional products include a four channel time-to-digital converter with histogram memory, and $20 \mathrm{MHz}$ position sensitive detectors with position encoding electronics.

\section{Janis Research Company, Inc. $\$ 506$}

P.O. Box 696

Two Jewel Drive

Wilmington, MA 01887-0696

Tel: $978-657-8750$

Fax: 978-658-0349

E-mail: janis@janis.com

www.janis.com

Janis combines over 35 years of manufac-

turing experience with extensive engineering capabilities to provide cryogenic systems for all research applications. Janis offers closedcycle refrigerators, $4 \mathrm{~K}$ refrigerators, continuous flow and variable temperature cryostats superconducting magnet systems, detector cooling dewars, dilution refrigerators, Helium-3 cryostats, and custom designs to meet any specific requirements.

\section{IEOL \#301}

11 Dearborn Road

Peabody, MA 01960

Tel: 978-535-5900

Fax: 978-536-2205

E-mail: eod@ijeol.com

wwwj.jeol.com

A $200 \mathrm{kV} \mathrm{FEG}$ with better than $13 \mathrm{~nm}$ point resolution in STEM; a 4.0 degree K UHV STM; a UHV AFM/STM with non-contact, atomic resolution; a new ambient/envinonmental SPM with cold and hot stage; a new SEM with $1.5 \mathrm{~nm}$ resolution and a new, really intuitive user interface; all the other high performance, reliable instruments in the JEOL product line. Ask for more information.

\section{- Johnsen Ulitavac \#420}

\section{Mainway}

Burlington, Ontario L7M 1A8

Canada

Tel: 905-335-7022

Toll Free: 800-268-4980

Fax: 905-335-3506

E-mail: juvinfo@ultrahivac.com www.ultrahivac.com

Manufactures a complete line of vacuum products for $R \& D$, production and light sources including six (6) axis manipulators featuring maximum \pm 4.00 inch $X Y$ motion, heating/cooling stages with $10 \mathrm{~K}$ to $1500 \mathrm{~K}$ temperature range, ultra-long stroke linear motion translators, UHV XYZ translators with $500 \mathrm{lbs}$. payload capacity, $X Y Z$ stages for in situ analysis, 5x10E-11 Torr UHV chambers, beam line components, monochromators and a wide range of vacuum systems for surface analysis, semiconductor, optoelectronics and coating. We specialize in unique, one-of-a-kind projects.

- Keithley Instruments, Inc. \#508

28775 Aurora Road

Solon, OH 44139

Tel: 440-248-0400

Fax: 440-248-6168

E-mail: product_info@keithley.com

www.keithley.com

Keithley will display its line of sensitive test instrumentation designed for materials research applications. Keithley has added to its family of electrometers with the new Model 6514 Programmable Electrometer. The Model 6514 is ideal for applications that demand fast, precise measurements of low currents or high resistances. Also highlighted will be the Model $65 \mathrm{High}$ Resistivity measurement package, and the Model 2182 Nanovoltmeter

\section{Kluwer Acodemic Publishers \#1 19 \\ 101 Philip Drive \\ Norwell, MA 02061 \\ Tel: 781-871-6600 \\ Fax: 781-871-6528 \\ E-mail: kluwer@wkap.com www.wkap.nl}

Kluwer Academic Publishers (KAP) is a sector of the Wolters Kluwer publishing group and operates worldwide from offices in Dordrecht, Boston and London. KAP is active in many academic and professional fields, producing high-quality English-language books, journals, loose-leaf publications, and electronic publications. Scientists and professionals from all over the world hold our publications in high esteem. This is achieved by a combination of editorial quality, peerreviewed information, and publications tailored to the customer's needs. Worldwide, Kuwer Academic Publishers provides the latest, up-to-date, scientific and professional information $-a$ valuable asset for a publishing house geared towands meeting the challenges arising from the information age.

\section{Krotos Anatytical, Inc. \#606}

100 Red Schoolhouse Road

Building A

Chestnut Ridge, NY 10977

Tel: 914-426-6700

Fax: 914-426-6192

E-mail: jd@kratos.com

www.kratos.com

Kratos is the leading manufacturer of materials analysis instrumentation providing superior capabilities for surface chemical characterization, elemental analysis and crystalline phase analysis. Techriques utilized are XPS/ESCA, AES/SAM, XRF (wavelength disbursive and energy disbursive $x$-ray fluorescence spectroscopy) and XRD ( $x$-ray diffraction). Instruments range from compact/ benchtop systems to ultra high performance research grade instrumentation.

\section{- Kurt J. Lesker Company \#501}

1515 Worthington Avenue

Clairton PA $15025-2700$

Tel: $412-233-4200$

Toll Free: 800-245-1656

Fax: $412-233-4275$

E-mail: sales@lesker.com; international@ lesker.com

wwwleskercom

Worldwide distribution of linear \& circular Torus ${ }^{\circledR}$ sputtering magnetrons; e-beam, thermal and epitaxy sources; targets and materials of all purities; custom UHV chambers; electrical, gas/liquid, motion feedthroughs; and the AccuQuad ${ }^{\mathbb{R}}$ RGA. Also offering high vacuum and $U H V$ sample manipulation and surface science components.
Lodd Reseorch Industries, Inc. \#221

13 Dorset Lane

Williston, VT 05495

Tel: $802-878-6711$

Fax: 802-878-8074

E-mail: ladres@worldnet.att.net ladd.cc/

Specialties include:

- Apertures-All electron microscopes. Platinum, molybdenum strips and discs $5 \mu \mathrm{m}$ and above.

- Microholes-Slits/holes, various metals and sizes. Applications include $x$-ray, gas flow, ion beam, E-beam, light flow and solder droplets

- Equipment-Vacuum evaporators, sputter coaters, ultra sonic cleaners, etc.

- Supplies-Tweezers, specimen mounts, standards, precision tools, chemicals, photographic supplies, etc.

- Lake Shore Cryotronics, Irx. \#510 575 McCorkle Blvd.

Westerville, $\mathrm{OH}$ 43082-8888

Tel: 614-891-2243

Fax: 614-891-1392

E-mail: sales@lakeshore.com www.lakeshore.com

Integrated Hall effect and magnetoresistance semiconductor material characterization systems with Van der Pauw resistivity measurements; features QMSA, a new datamanipulation algorithm which simultaneously derives concentrations and mobilities for multiple distributions of electrons and holes in layered semiconductor device structures. Also vibrating sample magnetometers, $\mathrm{AC}$ susceptometers / $\mathrm{DC}$ magnetometers, cryogenic platforms; high-level,

Windows ${ }^{\mathrm{TM}}$-based instrument management software; new Model 340 dual loop temperature controller with multiple sensor inputs, cryogenic temperature sensors, gaussmeters, Hall generators, electromagnets, and four-quadrant power supplies.

\section{Leica Microsystems Inc. \#616, 618}

111 Deer Lake Road

Deerfield, IL 60015

Tel: 847-405-0123

Toll Free: 800-248-0123

Fax: 847-405-0147

E-mail: infoleica-microsystems.com www.leica-microsystems.com Leica Microsystems will display EM sample preparation products including the new RES 100 ion beam etcher, the UCT ultramicrotome, and polycut/ultramiller. Leica will show the DM LM microscope, featuring flexibility, mechanical and thermal stability and infinity optics, as well as the $M Z$ FLIII stereofluorescence system. Leica's products for sample preparation, includin the RM 2165 fully automated microtome, will also be on display.

\section{Logitech Product Group \#421}

810 Sharon Drive

Westlake, $\mathrm{OH} 44145$

Tel: 440-871-0071

Fax: $440-871-8188$

E-mail: info@logitech-us.com

www.logitech-us.com

Logitech is a manufacturer of high precision sawing lapping and polishing equipment. Research and small-scale production systems are available for substrate backthinning and polishing GaAs, InP, saphire, silicon and other semiconductor wafers, electro-optic crystals, ceramics, waveguides laser rods and optical components. We also supply a full range of lapping and polishing consumables. For additional information call Keith Torrance at 800-321-5834.

LUXTRON Corporation \#320

2775 Northwestem Parkway

Santa Clara, CA 95051-0941

Tel: 408-727-1600

Fax: 408-727-1677

E-mail: maria@luxtron.com

www.luxtron.com

LUXTRON Corporation develops, manufactures, and markets unique sensors and instrumentation, based on optical and heat flow technologies, for a wide range of semiconductor, medical, industrial, and $R \& D$ applications.

\section{MBRAUN, Inc. \#202}

65 Parker Street, Unit 5

Newburyport, MA 01950

Tel: 978-462-1770

Fax: 978-462-1862

E-mail: mbgloveboxes@msn.com www.mbraun.com

MBRAUN produces inert atmosphere glove boxes (UNIlab, Labmaster, MB150BGI), isolators, welding chambers, production glove box lines, and a position sensitive detector for $x$-ray diffraction. Leading the industry in technology, advancements and simplicity as 
- MKS Instruments, Inc. \#423

Six Shattuck Road

Andover, MA 01810

Tel: 978-975-2350

Fax: 978-975-0093

E-mail: mks@mksinst.com

www.mksinst.com

MKS Instruments, Inc will feature several products across its entire product line oriented towards advanced materials research, such as low- and high-K dielectrics and copper manufacturing processes. These products represent the latest in measurement and control technology as well as in situ process diagnostics.

\section{- MMR Technologies, Inc. \#503}

1400 N. Shoreline Blvd., Suite A-5

Mountain View, CA 94043-1346

Tel: 650-962-9620

Fax: 650-962-9647

E-mail: mmr@mmr.com www.mmr.com

MMR Technologies manufactures temperature controlled systems — cryogenic cooling systems and wide temperature range thermal stages - which find application in materials research in electrical engineering physics, biology and chemistry applications over the temperature range of $10 \mathrm{~K}$ to $730 \mathrm{~K}$. They are also used in the cooling of computer chips, electronic devices, laser diodes, and thermal imaging devices, and in the characterization of the performance and properties of such devices as a function of temperature.

- Molecular Simulations, Inc. \#401,403

9685 Scranton Road

San Diego, CA 92121

Tel: 619-458-9990

Fax: 619-458-013

www.msi.com

Visit MSI to find out how computer modeling predicts properties, designs and characterizes new materials, and helps you to optmize processes. Application areas include electronics, where researchers study defect structures, interpret analytical data and understand chemical vapor deposition; and catalysis, where MSI's consortium develops technologies to understand reactions in zeolites and at surfaces. See how MSI will make simulation available on the Web.

\section{Corporation \#621}

5327 lacuzzi Street, Unit 3G

Richmond, CA 94804

Tel: $510-525-3070$

Fax: $510-525-4728$

E-mail: mtixpj@aol.com

www.mticrystal.com

Manufacturer of various single crystal substrates for photoelectronics, III-V nitrides, compound semiconductors and superconductor thin films. Major products include $\mathrm{MgAl}_{2} \mathrm{O}_{4}, \mathrm{Al}_{2} \mathrm{O}_{3}, \mathrm{BaTiO}_{3}, \mathrm{LaAlO}_{3}, \mathrm{SrTiO}_{3}$, $\mathrm{SrLaAlO}_{4}$ GaP, GaAs, GaSb, InP, etc. crystals and substrates. MTI also supplies sputtering targets, ceramic substrates and crucibles, high purity rane earth oxides and high quality 20 to $100 \mathrm{~nm}$ nanophase powders, including $\mathrm{SiC}, \mathrm{Si}_{3} \mathrm{~N}_{4} \mathrm{Si} / \mathrm{C} / \mathrm{N}$ and $\mathrm{Si}$.

\section{- MTS Systems Corporation \$307, 309}

14000 Technology Drive

Eden Prairie, MN 55344-2290

Tel: 800-944-1687

Fax: 800-777-1515

E-mail: info@mts.com

www.mts.com

MTS Systems Corporation designs, engineers and manufactures testing systems that characterize the mechanical properties of materials and structures. With the acquisition of Nano Instruments, Inc.-renamed the Nano Instruments Innovation CenterMTS provides nanometer and microNewton capabilities through Nano Indenter ${ }^{*}$ systems, in addition to microforce capabilities through the Tytron ${ }^{\mathrm{TM}} 250$ system, and lowforce capabilities through electromechanical and servohydraulic systems.
MVSystems, Inc. \#205

17301 W. Colfax Avenue

Suite 305

Golden, CO 80401

Tel: $303-271-9907$

Fax: 303-271-977

MVSystems, Inc. provides ultra high vacuum, multi-chamber PECVD/sputtering systems, arranged in a cluster tool configuration, specifically designed for thin-film semiconductor materials and devices. As part of the system sale, MVS guarantees, contractually, the opto-electronics properties of thin-film semiconductors, dielectrics and state-of-the-art electronic device performance for solar cells, thin-film transisto (for displays) and image sensors, etc. MVSystems, Inc. also provides hot wire CVD and electron beam systems.

Nono Instruments Innovation Center

1001 Larson Drive

Oak Ridge, TN 37830

Tel: 423-481-8451

Fax: 423-481-8455

E-mail: nano@nanoinst.com

www.nanoinst.com

See description under MTS Systems Corporation

- National Electrostatics Corporation \#305 P.O. Box 620310

7540 Graber Road

Middleton, WI 53562-0310

Tel: 608-831-7600

Fax: 608-256-4103

E-mail: nec@well.com

www.pelletron.com

National Electrostatics Corporation manufactures a wide variety of ion beam systems from below $100 \mathrm{keV}$ to the hundreds of $\mathrm{MeV}$ region. These systems include complete materials analysis systems using $\mathrm{MeV}$ ion beams including RBS, PDXE, NRA, ERD and AMS NEC also manufactures a wide range of beam handling and vacuum components including vacuum isolators for vacuum pumps.

\section{National High Mognetic Field Loboratory} (NHMFL) \#620,622

1800 E. Paul Dirac Drive

Tallahassee, FL 32310

Tel: 850-644-6257

Fax: 850-644-0534

E-mail: johnson@magnet.fsu.edu www.magnet.fsu.edu

The NHMFL provides unique facilities for probing the properties of materials in magnetic fields to $33 \mathrm{~T}$ (steady), $60 \mathrm{~T}(100 \mathrm{~ms})$, and $70 \mathrm{~T}(20 \mathrm{~ms})$ at no charge to qualified users. Researchers may probe the optical, magnetic, NMR, tensile, thermal and electrical properties of materials at temperatures from 800 microkelvin to $600 \mathrm{~K}$, and pressures to $14 \mathrm{MPa}$. Financial support is available for first-time users and long-term visitors.

\section{- Neocero, Inc. \#517}

10000 Vinginia Manor Road, Suite 300 Beltsville, MD 20705-4215

Tel: $301-210-1010$

Fax: 301-210-1042

E-mail: troyeconeocera.com www.neocera.com

Neocera, Inc. is a microelectronics and sensor-based technology company with leading edge materials expertise in thin film development and production. Neocera products include pulsed laser deposition systems, metal oxide thin films and thin film devices, cryogenic temperature controllers, cryogenic temperature sensors and HTS SQUID microscopy and microwave microscopy systems.
New Wove Research $\$ 110$

495 Mercury Drive

Sunnyvale, CA 94086

Tel: 408-328-0220

Fax: 408-328-0234

E-mail: lasers@new-wave.com

www.new-wave.com

New Wave Research manufactures and

delivers complete compact, high performance Nd:YAG laser micromachining systems. The QuikLaze operates at repetition rates up to $40 \mathrm{~Hz}$, with multiple wavelengths easily available from the infrared to the ultraviolet. Applications include marking, failure analysis, resistor trimming, LCD repair, material processing micromachining and your application.

\section{Nor-Cal Products, Inc. \#115 \\ P.O. Box 518 \\ 1967 S. Oregon Street \\ Yreka, CA 96097 \\ Tel: $530-842-4457$ \\ Toll Free: $800-824-4166$ \\ Fax: $530-842-9130$ \\ E-mail: ncsales@n-c.com}

www.n-c.com

Manufacturer of stainless steel vacuum components for over 35 years. Standard products include: NW, ISO, ASA, CF, and wire seal flanges; fittings, viewports feedthroughs and flexible hoses; manual and pneumatically actuated valves; heater jackets; liquid nitrogen, molecular sieve, water-cooled, and particulate foreline traps: and high vacuum and UHV manipulators. Custom chambers, manifolds, feedthrough collars and baseplates can be manufactured from customer specifications, sketches or drawings.

\section{NORAN Insinuments Inc. $\$ 609$}

2551 West Beltline Highway

Middleton, WI 53562

Tel: 608-831-6511

Fax: 608-836-7224

E-mail: info@noran.com

www.noran.com

NORAN Instruments manufactures premium systems for microscopy applications, and provides worldwide customer support for these instruments. For $x$-ray microanalysis, NORAN Instruments offers the new PC-based VANTAGE Digital Microanalysis System, the powerful VOY AGER Microanalysis System, and the Kevex high-value SIGMA Microanalysis System featuring SuperDry electronically cooled $x$-ray detectors with SuperQuantum windows. NORAN also offers a full line of WDS and electron diffraction products for SEM applications. For optical microscopy, NORAN Instruments offers the versatile $O$ Confocal Laser Scanning Microscope.

\section{- Omicron Associotes \$504}

1226 Stoltz Road

Bethel Park, PA 15102

Tel: 412-831-2262

Fax: 412-831-9828

E-mail: omiassoc@sgi.net

www.omicron-instruments.com

Omicron Vakuumphysik GmbH is a pri-

vately owned, independent German-based company specializing in the design and manufacture of a wide range of analytical research tools designed to be used in ultra high vacuum environments. Products include the world's most comprehensive range of UHV scanning probe microscopes, photoelectron spectrometers for XPS/UPS/ISS/AES, photoemission electron microscopes, low energy electron diffraction optics, Auger spectrometers, UHV evaporators, electron guns/ion beam sources and a series of complete multi-technique systems. To compliment the range of UHV SPMs and to meet new nano-technology applications, an ambient pressure scanning near-field optical microscope using

SPM technology (TtuinSNOM) was launched in 1998. Heavy investment in product development is a priority and twohirds of the staff in the international headquarters are physics or chemistry graduates with advanced degrees. The company was founded in 1984 and currently has approximately 120 employees worldwide with representation in 12 countries.

\section{Oxford Applied Research \#521}

Crawley Mill, Witney

United Kingdom

Tel: 44-1993-773575

Fax: 44-1993-702326

E-mail: sales@oxfordar.demon.co.uk www.oaresearch.co.uk

Manufacturer of growth, processing and characterization equipment for semiconductor or thin film research including RF atom sources for high-quality oxide/nitride growth and effective $\mathrm{H}$-atom precleaning of sensitive substrates. Also supplies e-beam evaporators, ECl nanocluster deposition sources for contact metallization or nanofabrication, ion sources for sputter deposition/cleaning and electron guns.

Oxford Instruments Americo, Inc. \#516 
- Ted Pella, Inc. \#319

P.O. Box 492477

4595 Mountain Lakes Blvd.

Redding, CA 96049-2477

Tel: $530-243-2200$

Fax: 530-243-3761

E-mail: sales@tedpella.com

www.tedpella.com

Ted Pella, Inc, is a major supplier of acces

sories and instruments for electron

microscopy. Our catalog and web site contain nearly 4,000 items including coaters, sputter coaters and a carbon coater for SEM applications, a wide range of calibration standards, photographic supplies, vacuum desiccators, and a complete digital imaging system.

Perkin-Elmer Corporation \#322

761 Main Avenue

Norwalk CT 06859

Tel: 203-762-1000

Fax: 203-762-6000

E-mail: info@erkin-elmer.com

www.perkin-elmer.com

The LAMBDA 900 is the ideal tool for research, design, manufacturing and $O C$ where UV/VIS/NIR spectroscopy is the technique of choice. Characterization of thin film coatings, mirrors, high performance optics and many other applications can be accomplished with one instrument. Enhanced UVWINLAB software provides a simple but powerful user interface for the LAMBDA 900.

\section{- Philips Analytical \#201 \\ 85 McKee Drive \\ Mahwah, NJ 07430 \\ Tel: 201-529-6111 \\ Fax: 201-529-5084 \\ E-mail: jennifer.gati@philips.con \\ www.analytical.philips.com}

Philips Analytical is the leading manufacturer of $x$-ray diffraction and $x$-ray fluonescence equipment as well as wafer analyzing and photoluminescence mapping systems. Information is available on all product lines. Philips Analytical is ISO 9001 certified. We adhere to the most stringent of the three ISO classifications which requires an established, effective quality system be in place.

Philips Electron Optics

(See FEI Company/Philips Electron Optics)

Photometrics

(See Roper Scientific)

- Physical Electronics $\$ 406$

6509 Flying Cloud Drive

Eden Prairie, MN 55344

Tel: 612-828-6100

Fax: $612-828-6322$

E-mail: marketing@phi.com

www.phi.com

Physical Electronics (PHI) develops, manu-

factures and markets surface analysis

instrumentation to perform auger electron

spectroscopy (AES), $x$-ray photoelectron

spectroscopy (XPS), secondary ion mass

spectrometry (SIMS) and time-of-flight

SIMS. These techniques characterize the top few atomic layers of a surface, providing spatially resolved information about elemental composition, chemical bonding and molecular structure.

\section{PIEZOMAX Technologies, Inc. \#204}

565 Science Drive

Madison, WI 53711

Tel: 608-238-4850

Fax: 608-238-5120

E-mail: mackay@piezomax.com piezomax.com

PIEZOMAX Technologies, Inc. designs and manufactures affordable, custom-designed flexure-guided nanopositioning stages for ultra-high precision motion at extremely high frequencies. Applications include semiconductor integrated circuit manufacture, magnetic disk drives, optical positioning devices, precision machining, nanoscale metrology and materials characterization. Call 608-2384850 or reference http://piezomax.com.
Princeton Instruments

(See Roper Scientific)

Princeton Scientific Corp. \$206

P.O. Box 143

Princeton, N] 08542

Tel: 609-924-3011

Fax: 609-924-3018

E-mail: psc1@IDT.com

www.princesci.com

Princeton Scientific Corp. offers various metal and oxide single crystals, like W, Mo, $\mathrm{Au}, \mathrm{SrTiO}_{3}, \mathrm{TiO}_{2}$ and $\mathrm{MgO}$, as well as a number of III-V materials such as GaAs, $\mathrm{GaP}$, InSb, InAs, InP, and mone. Cutting and polishing services are also provided. Also on display is a precision wire saw which allows for a very accurate cutting process and yields surfaces with almost 'lapped' quality.

\section{- Quad Group, Inc. \#605}

1815 South Lewis Stree

Spokane, WA 99224

Tel: $509-458-4558$

Fax: 509-458-4555

E-mail: quadgroup@spokane.net

www.spokane.net/quadgroup

A Complete Material Science Test Lab (and

Coatings Lab) in a Box... Romulus III-A executes tensile stress/strain (eng. and true), or in seconds switches to flexural modulus of rupture (3-point or 4-point loading) with automatic operation and parameter reporting-no operator judgment or errors. For the coating test lab, execute optional stud pull adhesion, shear, peel, tear, blade cutting, scratch, for electronics, functional coatings, or disk and head coatings. AND, micro-range tests for multiple micro electronic applications. NEW, our instrumented indentation tester, Alexandra I measures hardness, Young's Modulus, yield strength and strain hardening in minutes. Uses proven Hertzian concepts and simple polished metallurgical sample prep.

Excellent for characterizing all material

above 5 micrometers, and is valuable in

determining the properties as a function of depth, such as with IBMM and composites. Wonderful teaching lecture aid.

\section{Quolitou \#306}

575 Maude Court

Sunnyvale, CA 94086

Tel: $408-522-9200$

Fax: 408-522-8110

E-mail: sales@qualitau.com

www.qualitau.com

Qualitau, a leading supplier of reliability test equipment, is once again leading the industry in its development of new products. In 1997, we were first to develop electromigration test systems for copper metallization. New for 1999 is a test system designed for pulsed DC electromigration named the ACE. Please stop by our booth at MRS to learn mone about the complete line of reliability test systems for electromigration, hot carrier and TDDB.

\section{Quesant Instrument Corporation \#419 www.saesgetters.com \\ SAES Pure Gas is the manufacturer and}

29397 Agoura Road, Suite 104

Agoura Hills, CA 91301

Tel: $818-597-0311$

Fax: 818-991-5490

E-mail: qsales@quesant.com

wrww.quesant.com

Quesant produces easy-to-use, innovative and affordable scanning probe microscopes. Q-Scope ${ }^{\mathrm{M} M}$ SPMs offer multiple scanning modes and include innovative features such as our patented Isotopic Focal System ${ }^{\mathrm{TM}}$ fast scanning (up to 10 times faster than ather SPMs), and Windows-based software. All at prices that make sense.

\section{Renishow Inc. \#607}

623 Cooper Court

Schaumburg, IL 60173

Tel: 847-843-3666

Fax: 847-843-1744

E-mail: renmktsves@aol.com

www.renishaw.com

Products: Ramascope, the revolutionary Raman microscope for Raman and photoluminescence. Features include rapid measurement (typically $<10 \mathrm{~s}), 1 \mu \mathrm{m}$ lateral by $2 \mu \mathrm{m}$ depth resolution, $1.5 \mathrm{~cm}^{-1}$ spectra resolution, measurement range $240 \mathrm{~nm}$ to $1100 \mathrm{~nm}$, Renishaw proprietary extended scan facility, direct 2-D imaging, fiber probes, $x y z 0$ mapping and GRAMS $/ 32^{\mathrm{TM}}$ software. Accessories for variable tempera. ture $4 \mathrm{~K}$ to $1500^{\circ} \mathrm{C}$, high pressure and electrochemical measurements are also available.

\section{Research and PVD Materials Corporation} \#415

P.O. Box 4796

Wayne, NJ 07474

Fax: $973-575-6460$

Research and PVD Materials Corporation

manufactures a wide variety of highly characterized, high purity materials for the diverse and sophisticated requirements of the semiconductor, electronics, electro-optic and related research communities.

Products from this single-quality source include but are not limited to sputtering targets, thin-film deposition materials, fabricated forms of specialty and exotic metals, alloys, ceramics, intermetallics, custom fabrications and "one off" components.

\section{Rigaku/USA, Inc. \#509}

199 Rosewood Drive

Danvers, MA 0192

Tel: 978-777-2446

Fax: 978-777-3594

E-mail: info@rigaku.com

www.rigaku.com

Rigaku provides a wide range of $\mathrm{x}$-ray diffraction and fluonescence systems for the materials and semiconductor industries.

Rigaku is the leading supplier of wafer/disk analyzers worldwide. Rigaku has been one of the leaders in $x$-ray analytical instrumentation for more than 50 years and continues its leading edge in $x$-ray analysis.

\section{Roper Scientific Trenton \#316}

3660 Quakerbridge Road

Trenton, N] 08619

Tel: 609-587-9797

Fax: 609-587-8970

E-mail: postmaster@roperscientific.com

www.roperscientific.com

Roper Scientific, formed in April 1998 by the urion of Princeton Instruments and Photometrics, is the world's leading manufacturer of low light level scientific CCD and gated intensified cameras. Systems for spectroscopy or imaging are available that can detect in the NIR, visible, UV, or $x$-ray wavelength ranges.

\section{SAES Pure Gas, Inc. \#209}

4175 Santa Fe Road

San Luis Obispo, CA 93401

Tel: 805-541-9299

Fax: 805-541-9399

E-mail: jennifer_sawyer@saes-group.com worldwide distributor of UHP gas purifiers including the $G C 50$ gas purifier, MicroTor ambient temperature gas purifiers MonoTorn point-of-use gas purifiers MegaTor ${ }^{3}$ area/house purifiers, and the InsiTor ${ }^{8}$ Fast Pump. SAES also offers a wide range of analytical equipment and services.

\section{Sagitta Lid. \#114}

4 Hayetzira Street

Ramat-Gan 52521

Israel

Tel: 972-3-7514601

Fax: 972-3-7514607

E-mail: info@sagitta.co.il

Sagitta's NEXT-1 cross-sectioning system is designed to meet the challenges of semiconductor sample preparation needs.

Featuring an entirely new and fully automated solution, the system is capable of polishing samples "straight to the point" NEXT-1, a Windows-NT based system, incorporates advanced optics and Sagitta's

proprietary sub micron polishing technology. The system enables easy target positioning, target definition, self-controlled polishing and superior process monitoring capabilities. Featuring 0.1-micrometer accuracy, NEXT-1 is the only tool for polishing samples to a predefined point or polishing along a specified line.

Scivision \#116

200 Wheeler Road

Burlington, MA 01803

Tel: $781-272-4949$

Fax: 781-272-6868

www scivis 
Scen Instruments \#218

A Division of Materials Research Group, Inc.

12441 West 49th Avenue, Suite 3

Wheat Ridge, CO 80033-1927

Tel: $303-425-6688$

Fax: 303-425-6562

E-mail: soeninstr@aol.com

www.matresgrp.com

Soen Instruments specializes in quality con-

trol software for the semiconductor and

photovoltaic industry. Our characterization systems were designed to measure the quantum efficiency, current-voltage, spectral response, conductivity and thickness as well as microscopic viewing of thin films. Our systems provide measurements that are fast, accurate, reliable, low-maintenance, cost competitive and can be customized to meet your specifications.

\section{South Boy Technology, Inc. $\# 500$ \\ 1120 Via Callejon \\ San Clemente, CA 92673 \\ Tel: $949-492-2600$ \\ Toll Free: $800-728-2233$ \\ Fax: 949-492-1499 \\ E-mail: sbt@southbaytech.com}

www.southbaytech.com

South Bay Technology will be exhibiting

the industry's most advanced sample

preparation systems and supplies.

Featured products include:

- PC150 Plasma Cleaner for TEM samples and sample holders

- IV3 Ion Milling System with new low energy gun technology

- RIE200 Reactive Ion Etcher

- PE150 Plasma Etcher

- 900 Series lapping and polishing systems

- Systems for orienting, cutting and

polishing single crystals

\section{SPECS USA, inc. \#418}

Surface Analysis Division

635 South Orange Avenue

Sarasota, FL 34236

Tel: $941-362-4877$

Fax: $941-364-9706$

E-mail: support@specs-usa.com www.specs.de

SPECS will present its new high performance UHV surface analysis components at the MRS Spring Meeting which includes: high performance, low energy electron diffraction optics, Er-LEED 100/150; high resolution electron energy loss spectrometer. DELTA 0.5; and the ultra-high density plasma UV source, UVS 300 . SPECS, with over 15 years of experience in the field of ultrahigh vacuum products, is a leading manufacturer of surface analysis components and systems for XPS, UPS, ISS, AES, SAM SEM LEED, EELS, SIMS and SNMS. Contact us for additional information.

\section{- Springer-Verlag New York, Inc. \#121}

175 Fifth Avenue

New York, NY 10010

Toll Free: 800-SPRINGER

Fax: 201-533-3503

E-mail: custserv@springer-ny.com www.springer-ny.com

Visit our booth to see new titles such as

Computational Materials Science,

Macromolecular Science and Engineering, Cluster Beam Symthesis of Nanostructured Materials, Nanotechnology, and Cellular Neural Netuorks. These and last year's best-selling titles (e.g., Ferroelectric Phenomena in Crystals, Quantum Dots, and Understanding Materials Science) are available at a $20 \%$ discount. The Physics Sale 1999 is also in progress (nearly 200 titles at up to $65 \%$ discount).

\section{- STAIB Instruments \#602}

813 Diligence Drive, Suite121E Newport News, VA 23606-4285 Tel: $757-873-0099$

Fax: $757-873.0130$

E-mail: staib-us@staib-instruments.com www.staib-instruments.com Manufacturers of compact, high-perfor mance electron-optical equipment for in situ material analysis, including: RHEED sysems and RHEED energy analyzer, with data acquisition, to study structure and quality of thin films; photo-emission electron microscopes (PEEM), for dynamic studies of chemical distribution with high time and space resolution; energy analyzers for AES, XPS and UPS; and electron guns for analytical surface studies. Visit our web site at www.staib-instruments.com.

Superior MicroPowders, UC \#102

3740 Hawkins NE

Albuquerque, NM 87109

Tel: 505-342-1492

Fax: 505-342-2168

E-mail: caruso01@smpi.com

www.smpi.com

Superior MicroPowders (SMP) is a manufacturer of advanced powders and powderbased materials for energy, medical and thick-film applications including a variety of luminescent powders for advanced display applications. SMP utilizes a proprietary, non-material specific powder manufacturing process. SMP produces powder that are non-milled, spherical and unagglomerated with controlled microstructure. The powders have small tailorable particle sizes in the 1-2 micron size range with controlled particle size distributions. SMP's powders are ideally suited for more advanced powder deposition methods and organic-based delivery systems, which allow better control over paste rheology and deposited features.

\section{- Sufface/Interface, Inc. \#615 \\ 260 Santa Ana Court trometers reconding reflectance and trans- mittance of the layer stacks simultaneously}

Sunnyvale, CA 94086

Tel: 408-732-7111

Fax: 408-732-7191

E-mail: sii@surfaceinterface.com

www.surfaceinterface.com

Surface/Interface is a privately held firm specializing in providing innovative products for OEM, production facility and

$R \& D /$ university customers in the semiconductor media, sputter deposition and R\&-D markets. The firm has operating groups focused on metrology for $C D$ measurement and vacuum products for sample transfer and positioning. The Vacuum Products Group's core expertise is in the handling, transporting, positioning and manipulation of samples. Semiconductor wafers, substrates, flat panels and other materials are handled with precision and reliability in UHV, HV, ultra-clean, ultra-dry and other challenging controlled environments. The product line includes MESC-compatible wafer transport systems.

\section{- SVT Associates, Inc. \#523}

7620 Executive Drive

Eden Prairie, MN 55344

Tel: 612-934-2100

Fax: 612-934-2737

E-mail: svta@svta.com

www.svta.com

SVT Associates, Inc. offers a full range of thin film deposition and process monitoring components and systems including MBE systems for III-V, II-VI and SiGe

UHV/CVD for SiGe production; SiC CVD

and bulk growth; metal/oxide CVD; and

UHV sputtering. Newly developed

A1GaInN MBE systems guarantee material specifications. Components include RF

plasma and thermal effusion cells. Contact

us for nitride epi-service.

\section{Toylor \& Fronics \#118}

325 Chestnut Street

Philadelphia, PA 19106

Tel: $215-625-8900$

Fax: 215-625-2940

E-mail: pkilgarriff@taylorandfrancis.com www taylorandfrancis.com

Taylor \& Francis has been an international publisher of scientific works for over 200 years. Stop by our booth to check out our books Liquid Crystals in Complex Geometries
Formed by Polymer and Porous Networks, The Optics of Thermotropic Liquid Crystals, MicroOptics, High-Temperature Superconductors and Other Superfluids, and Antioxidative Stabilization of Polymers, as well as our journals Liquid Crystals, Molecular Physics, Journal of Modem Optics, Philosophical Magazine Parts A, B, and Letters, and International Reviews in Physical Chemistry.

Technical Instrument San Francisco \#103 348 Sixth Street

San Francisco, CA 94103-4733

Tel: 415-431-8231

Fax: 415-431-6491

www.techinst.com

Scanning probe instruments and confocal microscopes: real-time confocal scanning optical microscopes and retrofit attachments. TISF has a confocal integrated with a scanning probe microscope. 2D and 3D software available as an option.

\section{Theiss Hard-and Software \#117}

Dr-Bernhard-Klein-Str. 110

D-52078 Aachen

Germany

Tel: 49-241-5661390

Fax: 49-241-9529100

E-mail: theiss@mtheiss.com

www.mtheiss.com

M. Theiss Hard- and Software offers software and hardware for optical spectroscopy. The focus is on thin film analysis and design. Spectrum simulation programs for research, design and production control ane developed which may be coupled to spec-

- Thermionics Vocuum Products \#409, 411 231-B Otto Street

Port Townsend, WA 98368

Tel: $800-962-2310$

Fax: 360-385-6617

E-mail: sales@thermionics.com

www.thermionics.com

Thermionics manufactures vacuum components and systems including: jon pumps, sample handling and transfer devices, RNN'M differentially pumped motary seals, Clearview ${ }^{\mathrm{TM}}$ heated viewports; $3-20 \mathrm{~kW}$ e-Gun ${ }^{\mathrm{TM}}$ evaporation sources; maTChed ${ }^{\mathrm{TM}}$ thermocouple gauges, PyraFlat ${ }^{\mathrm{TM}}$ flanges, fittings, feedthroughs, hardware; PVD, PLD and custom systems; RHEED; DRS-1000 ${ }^{\mathrm{TM}}$ in situ, remote substrate temperature measurement/control system.

ThermoMicroscopes \#308, 311

1171 Borregas Avenue

Sunnyvale, CA 94089-1306

Tel: 408-747-1600

Fax: 408-747-1601

E-mail: info@thermomicro.com

www.thermomicro.com

ThermoMicroscopes is a result of the merg

er between Park Scientific Instruments and TopoMetrix. The new company is now the premier supplier of atomic force and scanning probe microscopes. ThermoMicroscopes now offers instruments that operate in all environments (ambient, liquid and UHV) for research and industrial use. Stop by our booth for more information.

\section{TP., Inc. \$619}

Advanced Technologies

3921 Academy Parkway North NE

Albuquerque, NM 87109-4416

Tel: 505-342-4448

Fax: 505-345-8155

E-mail: yspoonerotplinc.com

www.tplinc.com

TPL, Inc., Albuquerque, NM produces electronic materials and nondestructive test instrumentation. The Porotec ${ }^{\text {M }}$ Thin Film Porosimeter is the only instrument capable of providing full BET and BJH analyses of thin films in their deposited forms. TPL produces $50 \mathrm{~nm}$, capacitor- grade barium sublimation pumps; XYZ manipulators,

be customized for specific applications.

Union Carbide Crystal Products \#522

750 South 32nd Street

Washougal, WA 98671

Tel: $360-835-2001$

Fax: 360-835-9848

Crystal Products, located in the Pacific

Northwest, offers Czochralski-grown sapphire substrates for blue LED, superconductors, and SOI, radiation-hardened, IC devices. Sizes are 2 -in. and 3 -in. diameter, C-plane; 2-in. to 6-in. diameter, R-plane; and SOS epitaxial wafers. See our Sapphire Research Kits containing substrates oriented to $A, R \& C$ axes. 